
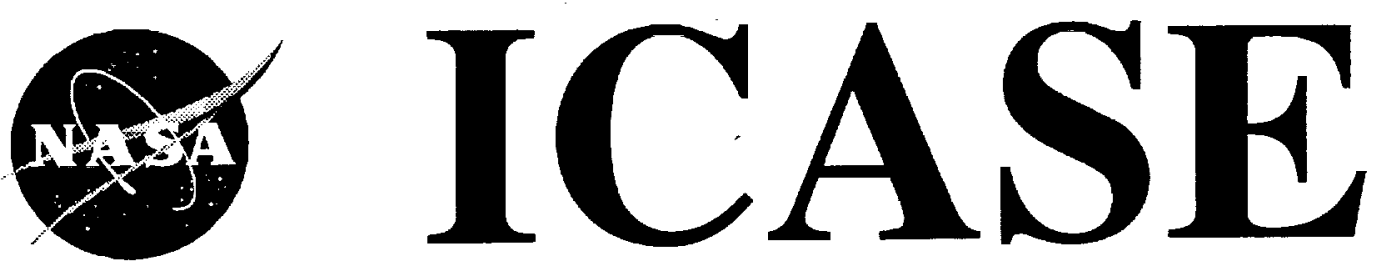

\title{
A PERSPECTIVE ON UNSTRUCTURED GRID FLOW \\ SOLVERS
}

\section{Venkatakrishnan \\ V. Venkatakrishman}

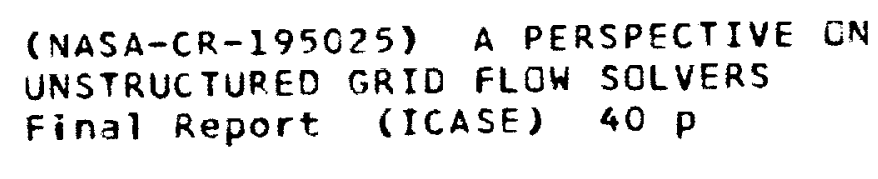

Final Report (ICASE) $40 \mathrm{p}$

N95-22931

Unclas

G3/64 0042549

Contract NAS1 - $1948 \underline{\underline{\underline{\underline{0}}}}$

February 1995

Institute for Computer Applications in Science and Engineering NASA Langley Research Center

Hampton, VA 23681-0001

SRA Operated by Universities Space Research Association 
$-$ 


\title{
A PERSPECTIVE ON UNSTRUCTURED GRID FLOW SOLVERS
}

\author{
V. Venkatakrishnan * \\ Institute for Computer Applications in Science and Engineering \\ MS 132C, NASA Langley Research Center \\ Hampton, VA 23681-0001
}

\begin{abstract}
This survey paper assesses the status of compressible Euler and Navier-Stokes solvers on unstructured grids. Different spatial and temporal discretization options for steady and unsteady flows are discussed. The integration of these components into an overall framework to solve practical problems is addressed. Issues such as grid adaptation, higher order methods, hybrid discretizations and parallel computing are briefly discussed. Finally, some outstanding issues and future research directions are presented.
\end{abstract}

*This research was supported under the NASA contract No. NAS1-19480 while the author was in residence at the Institute for Computer Applications in Science and Engineering (ICASE), NASA Langley Research Center, Hampton, VA 23681. 


\section{Introduction}

Computational Fluid Dynamics (CFD) has evolved rapidly as a discipline and is increasingly being used to complement the wind tunnel, especially in preliminary design [50]. Based largely on the mathematical foundations laid among others by Lax [88] and Godunov [52], the field has come into its own in the last decade. Great advances have been made in the areas of spatial discretization, grid generation and solution strategies. Tremendous advances in computer architecture and networking speeds have contributed to the field significantly as well. By the mid 80 's, many different groups around the world were able to compute three-dimensional flows over simple realistic aerodynamic configurations. The grids employed were of the body-fitted or structured grid type. One-dimensional models were extended to deal with multiple dimensions in a natural way because of the structure by using the so-called generalized coordinates [161]. However, the task of generating structured grids about complex configurations presented a serious challenge. The widely-used multi-block structured grid approach solves this problem by tessellating the domain between the body and the far-field into simple logically rectangular blocks, so that structured grids can be generated easily within each block [173, 81]. The automation of the blocking and the grid generation process are difficult tasks that are continually being refined. Another powerful approach uses overlapping or chimera grids $[19,110]$. Here, structured grids generated about the different components, are allowed to overlap. Automation of blocking, grid generation and the preprocessing required for deriving the interpolation operators are continually being improved.

The desire to compute flows over complex configurations also spawned a surge of activity in the area of unstructured grids. The term "unstructured grids" will be used primarily in this paper to mean grids composed of simplices, which are triangles in two dimensions and tetrahedra in three dimensions. Unstructured grids have always been used in finite element circles, but have become popular in the finite volume community only fairly recently. They provide flexibility for tessellating about complex geometries and for adapting to flow features, such as shocks and boundary layers. An underlying premise is that unstructured grid generation is far more automatable than are the tasks associated with multi-block structured grid generation.

It should be mentioned that the flow solver only constitutes a part of the overall solution methodology. Other tasks, such as grid generation, interfacing with geometry packages, visualization and post-processing are equally important, and demand considerable skills and resources. The outline of

this survey paper on unstructured grid flow solvers is as follows. Section 2 presents the governing equations in integral form. Section 3 reviews some popular finite volume spatial discretization schemes. Section 4 reviews the finite element approach for spatial discretization. Section 5 reviews the turbulence models in vogue for unstructured grid computations. Section 6 presents the various alternatives available for time-discretization for steady and unsteady problems. Section $\tau$ addresses the important topic of grid adaptation. Sections 8 and 9 examine higher order accurate schemes and hybrid discretizations. Section 10 briefly examines parallel computing issues. The paper concludes with a listing of issues to be resolved and some future research directions. In addition to the papers cited in this article, the reader is encouraged to read, especially regarding spatial discretization, the AGARD Report [1] on unstructured grid methods for advection dominated flows.

\section{Governing equations}

The equations governing compressible fluid flow in integral form for a control volume $\mathcal{V}(t)$ with boundary $\mathcal{S}(t)$ are given by

$$
\frac{\partial}{\partial t} \int_{\mathcal{V}(t)} W d v+\oint_{\mathcal{S}(t)}[F(\boldsymbol{W}, \boldsymbol{n}, \boldsymbol{s})-G(\boldsymbol{W}, \nabla W, \boldsymbol{n})] d a=0
$$

where

$$
W=[\rho, \rho \boldsymbol{V}, \rho e]^{T}
$$




$$
\begin{aligned}
F(W, n, s) & =(\boldsymbol{V}-\boldsymbol{s}) \cdot \boldsymbol{n} W \\
G(W, \nabla W, \boldsymbol{n}) & =[0, \boldsymbol{t}, \boldsymbol{t} \cdot \boldsymbol{V}-\boldsymbol{q} \cdot \boldsymbol{n}]^{T} \\
\boldsymbol{t} & =\boldsymbol{n} \cdot \overline{\bar{T}} \\
\overline{\bar{T}} & =[(-p+\lambda \Theta) \overline{\bar{I}}+2 \mu \overline{\bar{D}}]
\end{aligned}
$$

In the formulas given above $\rho$ is the density, $V$ is the velocity vector with Cartesian components $V_{i}, e$ is the specific total energy, $n$ is the outward unit normal vector of the boundary $\mathcal{S}(t)$ and $s$ is the velocity vector of the boundary. Also, $\mu$ is the molecular viscosity, $\lambda$ is the bulk viscosity related to $\mu$ by Stokes' hypothesis, $\lambda=-2 / 3 \mu, \overline{\bar{I}}$ is the identity tensor, $\overline{\bar{T}}$ is the stress tensor and $\overline{\bar{D}}$ is the deformation tensor given by

$$
D_{i j}=\frac{1}{2}\left(V_{i, j}+V_{j, i}\right)
$$

where $V_{i, j}$ denotes the partial derivative of the $i$ th component of $V$ with respect to the Cartesian coordinate $x_{j}$, i.e. $V_{i, j}=\frac{\partial V_{i}}{\partial x_{j}}$. $\Theta$ stands for the divergence of $V$ given by $V_{i, i}$ with the usual summation convention. $\boldsymbol{q}$ is the heat flux given by Fourier's law

$$
\boldsymbol{q}=-\hbar \nabla T
$$

where $K$ is the thermal conductivity of the fluid and $T$ is the temperature. These equations are augmented by the equation of state, which for a perfect gas is given by

$$
p=(\gamma-1)\left(\rho e-\frac{1}{2} \rho|V|^{2}\right)
$$

Eqn. (1) represents the conservation laws for the mass, momentum (the Navier-Stokes equations) and energy. It holds for any volume and in particular, holds for a specific volume associated with each grid point, termed the control volume.

\section{Finite volume spatial discretization}

It is assumed that a grid about the geometry of interest has been generated by some suitable method. The various grid generation techniques in use are reviewed in the survey papers by Thompson and Weatherill [166] and Mavriplis [106]. On a given grid, one has at least two choices as to where to locate the variables, giving rise to the cell-vertex and the cell-centered approaches. In the cell-vertex approach, the variables are stored at the vertices of the grid, whereas in the cell-centered approach they are stored at the centroids of the cells. There is yet another approach that stores only the averages associated with control volumes. If the scheme possesses second order spatial accuracy or less, this approach is no different than a cell-centered approach; the higher order scheme is covered briefly in Section 8.

The concept of using arbitrary control volumes to solve numerically the conservation laws was established by the late 70's, at least in theory [89]. Jameson and Mavriplis [73] reported some of the earliest results from solving the two-dimensional Euler equations on regular triangular grids that were obtained by subdividing quadrilateral grids. In a cell-centered setting, they extended much of what was established for finite volume schemes on structured grids $[74,67]$ to triangular grids. This included the constructions of central-difference-like approximation for the convective terms and a blend of dissipative terms to suppress odd-even decoupling and to capture discontinuities, and the incorporation of multigrid ideas. Solutions and convergence rates of comparable quality to structured grids were obtained. Second order accuracy was demonstrated by using a sequence of uniform grids. In 1986, Jameson et al. [72] presented their paper dealing with inviscid transonic flow over a complete aircraft. The paper's contributions included grid generation for complex geometries using the Delaunay triangulation approach and the development of a cell-vertex flow solver. The control volume for each vertex in the tetrahedral grid was taken to be the union of all tetrahedra sharing that vertex. They also showed 
the correspondence between this and a Galerkin finite element procedure with piecewise-linear basis functions. The nondissipative approximation was augmented with dissipative terms. In addition, the paper also presented a first discussion on a scheme with positive coefficients for triangles, although the proposed scheme was only first order accurate. Figure 1 shows the surface Mach contours for inviscid transonic flow over a Boeing $747-200$ taken from the paper of Jameson et al. [72]. The flow conditions are $M_{\infty}=0.8$ and $\alpha=2.73^{\circ}$. The mesh used for the calculation contained 12,038 nodes (5r914 tetrahedra) and the calculation took about 25 minutes on a Cray XMP computer. Since this seminal effort, tremendous advances have been made in grid generation and vectorization of the flow solver and are outlined in the sequel [71].

In 1973, Boris and Book [25] introduced the Flux Corrected Transport (FCT) scheme that converted a first order accurate monotone scheme to a higher order scheme by adding limited amounts of antidiffusive flux to prevent spurious oscillations. Harten [54] developed a mathematical formulation that gave rise to the Total Variation Diminishing (TVD) schemes which also added limited amounts of antidiffusive fluxes to a first order scheme to achieve monotonicity. Van Leer [169] independently devised the concept of limiting when designing monotonicity preserving schemes for conservation laws. He devised the Monotonic Upstream-centered Scheme for Conservation Laws (MUSCL) schemes which relied on a piecewise-polynomial reconstruction procedure that enforced monotonicity principles by using nonlinear functions called limiters. The jumps at the interfaces were resolved by using a Riemann solver, much like Godunov's scheme. Since the Riemann problem for the Euler equations requires an iterative procedure and is expensive, the quest was on for approximate Riemann solvers. Roe [142, 143] and Osher [120] constructed two such schemes that proved to be particularly good in concert with the MUSCL approach on structured grids. For an excellent discussion on the development of upwind schemes, see the text by Leveque [91]. By the mid 80 's, MUSCL schemes were being used by a number of groups to compute aerodynamic flows on structured grids $[164,160]$. These schemes were gradually being extended to deal with unstructured grids.

Desideri and Dervieux [40] devised cell-vertex finite volume schemes for unstructured grids using MUSCL ideas. Given pointwise values at the cell vertices of a triangulation, they employed a reconstruction procedure that made use of gradients in neighboring triangles. The flux was constructed using Osher's approximate Riemann solver. Limiters were used in a one-dimensional fashion but a multidimensional monotonicity principle was not satisfied. Nevertheless, respectable results were obtained for many inviscid computations. Löhner et al. [95] tested a FEM-FCT scheme for Euler and NavierStokes equations. Fezoui and Stoufflet [45] proposed and tested a class of implicit upwind schemes that utilized various upwind approximations. Whitaker et al. [185] constructed a similar scheme using Roe's flux-difference splitting scheme and obtained results for many transonic and supersonic flows. Venkatakrishnan and Barth [176] constructed an upwind scheme for cell-centered triangular grids that also employed MUSCL ideas as did Batina [16], Knight [84] and Frink [47]. However, none of these efforts really guaranteed the absence of oscillations in the multi-dimensional case.

Barth and Jespersen [15] made a radical departure from one-dimensional thinking for satisfying monotonicity principles. They enunciated a monotonicity principle in multiple dimensions similar to that employed by Van Leer [169] and Spekreijse for structured grids [160], namely that the reconstructed distribution in the control volume be bounded everywhere by the values of the neighbors (including the vertex representing the control volume) and satisfied the principle by constructing a truly multidimensional limiter. They employed Roe's approximate Riemann solver for the evolution phase. As an ultimate test, they computed oscillation-free solutions for transonic flow over an airfoil on a highly irregular mesh. They obtained solutions of comparable accuracy with both cell-centered and cell-vertex schemes. This MUSCL approach has been adopted since by other researchers $[5,7,51]$. The multidimensional limiter in [15] may be thought of as a generalization of the min-mod limiter and as such, leads to convergence difficulties. Venkatakrishnan [174] has analyzed this problem and has proposed modifications that ameliorate the situation at the expense of monotonicity. Aftosmis et al. [3] have found that the modifications proposed in [174] significantly improve the convergence as well as solution 
accuracy in test problems. Shu [153] has proposed the Total Variation Bounded (TVB) schemes which weaken the TVD property by allowing for small-scale oscillations although the motivation here is to avoid clipping smooth extrema. The effect of TVB modification on convergence has not been studied yet.

Frink et al. $[47,49]$ developed an upwind cell-centered three-dimensional flow solver. They employed a weighted averaging procedure that interpolated variables from the centers of tetrahedra to the vertices and used these vertex values to compute the gradients within each tetrahedron. These gradients were used to interpolate the variables to the centers of the faces of the tetrahedra. This was followed by the use of Roe's approximate Riemann solver. The reconstruction procedure was linear and therefore, not monotonicity preserving. Nevertheless, the averaging procedure seemed to introduce enough dissipation that good transonic flow solutions were obtained without using limiters. The reconstruction procedure has since been modified to be linearity-preserving [48] based on the work of Holmes and Connell [61]. Laminar viscous capability has been been added to this code. Figure 2a depicts the surface grid and "oil flow" pattern for laminar flow over a delta wing at $M_{\infty}=0.3, \alpha=20.5^{\circ}, R \epsilon_{L}=0.9 \times 10^{6}$. The flow has been computed by Frink using the methodology described in [48]. The grid containing 730,454 tetrahedral cells was generated by the Advancing-Layers Method [132] which produces thinlayer tetrahedral grids suitable for computing viscous flows. Evidence of primary, secondary and tertiary vortices may be seen in Figure $2 a$. Figure $2 b$ shows the pressure profiles at four chord stations and the comparisons with the results from the structured grid code CFL3D [164] and the experimental data of Hummel [66].

Barth $[11,12]$ presented extensions of the schemes of [15] to three-dimensional inviscid and viscous flow computations. The main contributions of these papers included the discretization of the viscous terms using a finite element procedure and the use of edge-based data structures for discretizing the inviscid and viscous terms. In addition, least-squares and data-weighted procedures for the construction of gradients were outlined. Mavriplis [102, 105, 101] developed an explicit vertex-based finite element multigrid scheme for the two- and three-dimensional Euler and Navier-Stokes equations. He also presented edge-based data structures. In $[11,105,98,97]$ a case is made for using cell-vertex approximations and edge-based data structures in three dimensions by examining the computational complexity and storage.

The issue of cell-vertex vs cell-centered approximations is still an open one, particularly in three dimensions. In two dimensions the ratio of the number of cells to the number of vertices is 2 whereas in three dimensions this ratio could be arbitrarily large, although it is typically between 5 and 6 for nice tetrahedralizations. In the case of tetrahedral grids, the flux computations in a cell-vertex scheme can be cast as loops over edges whereas in a cell-centered scheme they are loops over triangular faces. The ratio of the number of faces to the number of edges in a typical tetrahedral grid is roughly 2 . Therefore, it is true, as argued in $[11,105]$ that on a given grid, the cell-centered approximation incurs considerably more computational effort and memory requirements. However, there is some evidence that on a given grid, the solution quality of a cell-centered scheme is superior to that of a cell-vertex scheme [134]. This is more than likely due to the fact that for triangular/tetrahedral grids the control volumes in a cell-centered scheme are smaller than those in a cell-vertex scheme. It is not clear whether the cell-vertex scheme requires a grid that has as many vertices as the number of tetrahedra employed by a cell-centered scheme to achieve the same level of accuracy. If this is the case, the cell-centered scheme will prevail because it requires much smaller grids to be generated. However, it appears that a cell-vertex scheme is better suited to computing the viscous fluxes, especially when the triangulations become highly irregular. As discussed later in this section, the viscous terms in a cell-vertex scheme are typically discretized using a Galerkin finite element approach, whereas a finite-volume viewpoint is adopted in a cell-centered scheme. It has been shown [11,90] that a finite element discretization of a diffusion operator with linear basis functions obeys the discrete maximum principle if a Delaunay triangulation is used. This result is true only in two dimensions and does not hold in three dimensions. On the other hand, the finite volume discretization of the diffusion operator in a cell-centered setting 
does not guarantee a discrete maximum principle in two or three dimensions. It is interesting to note that the centroidal control volumes that are used with cell-vertex schemes are not necessarily convex and can assume rather odd shapes, especially in the case of highly stretched grids. One alternative is to use the Voronoi cells, which are guaranteed to be convex [86], but this approach requires care at the boundaries.

Significant developments have also taken place in the area of positive schemes. These require that the semi-discrete scheme be expressible in the form

$$
\frac{d u_{i}}{d t}=\sum_{j \in \mathcal{N}_{i}} C_{i j}\left(u_{j}-u_{i}\right),
$$

where the $C_{i j}$ 's are non-negative, and $\mathcal{N}_{i}$ denotes the set of neighbors of $i$. As observed in [72], if the $C_{i j}$ 's are constants, the scheme is linear and hence only first order accurate. Higher order versions of such schemes have been developed $[42,33,69,70]$. These may be regarded as generalizations of TVD schemes to conservation laws in multi-dimensions. Durlofsky et al. [42] have developed a cellcentered scheme that makes use of gradients in auxiliary triangles similar to the scheme of [176], but incorporates a multi-dimensional limiter to prevent oscillations. Both the schemes have the drawback of requiring nice triangulations, since the auxiliary gradients could be ill-defined on highly nonuniform meshes. Jameson [69] has developed a theory for Local Extremum Diminishing (LED) and Essentially Local Extremum Diminishing (ELED) schemes leading to Symmetric and Upstream Limited Positive (SLIP and USLIP) schemes. He has also presented an analysis for schemes to resolve shocks with one interior point [70]. A simple scheme that meets the conditions is termed a Convective Cipwind and Split Pressure (CUSP) scheme, which is closely related to the AUSM scheme of Steffen and Liou [92]. The schemes have been tested on structured grids for inviscid and viscous flows [162].

A different avenue to realizing upwinding on unstructured grids is provided by the fluctuationsplitting schemes $[39,121]$. In multi-dimensions, the weak link in the MUSCL formulation is the use of a directionally-split Riemann solver. This approach is routinely adopted, but can misinterpret features not aligned with the grid. To overcome this problem, various multi-dimensional Riemann solvers have been proposed in the literature $[38,125,148,146]$, but have not gained widespread acceptance. Rather than adopt the approach of reconstruction followed by an approximate Riemann solver to bring in the upwinding, fluctuation-splitting schemes consider the average time evolution of a complete cell (a triangle or a tetrahedron) with the unknowns located at its vertices, and then update the values at the vertices by the effect of linear wave solutions evolving the piecewise linear data over a cell. If the distribution formulas are unbiased, this leads to the well-known Ni's scheme [119], a centered scheme which requires the addition of dissipative terms. Several uwpind fluctuation-splitting schemes for scalar advection equation have been developed; these are compared in [121]. Extension of fluctuation-splitting schemes to systems of equations relies on the decomposition of flux divergence into scalar waves. The decomposition is not unique, and several strategies such as characteristic decomposition [38] and simple wave models $[144,125]$ have been investigated. Some of the advantages of the fluctuation-splitting approach are that they result in compact stencils and that they do not use ad-hoc dimensionally-split Riemann solvers. Some of the disadvantages include the fact that the scheme is only second order accurate at steady state, and that they bind the user to using a decomposition technique. Recently, a positive linearity-preserving fluctuation-splitting scheme has been constructed for the multi-dimensional scalar advection equation using limiters [155]. It differs from the other approaches in that a purely algebraic (not geometric) viewpoint is adopted. This approach has enabled Sidilkover [154] to devise a fluctuation-splitting scheme for the Euler equations that may be viewed as a genuine multi-dimensional extension of Roe's scheme without having to resort to decomposition into scalar waves. A unique property of this scheme is that a nonlinear Gauss Seidel procedure can be used as a relaxation scheme.

In finite volume schemes, a spatial (possibly monotonicity-preserving) approximation for the inviscid terms is combined with a centered approximation for the viscous fluxes. A basis for this "operatorsplitting" approach is furnished by Roe[145] who showed by means of elegant analysis in one dimension 
how a monotonicity-preserving discretization for the inviscid terms when combined with a centered approximation for the viscous terms suppresses spurious modes and also yields a nonoscillatory solution for all cell Reynolds numbers. On unstructured grids, the viscous fluxes are discretized by using either finite volume or finite element approaches. In the case of cell-centered discretizations Frink et al. [48] compute the first derivatives at the vertices of the triangulation, which are then averaged to obtain the viscous fluxes at the faces. In the case of cell-vertex schemes, the viscous terms are usually derived by adopting a Galerkin finite element approach $[14 \bar{\tau}, 11,101]$, although there is an equivalent finite volume interpretation. This formulation results in a compact stencil involving only the nearest neighbors, and also exactly reproduces the gradient of a linear function. A simpler formulation has been proposed in [98]. Here, the viscous fluxes are computed by averaging the cell-vertex gradients, which are available from the reconstruction procedure for the inviscid terms. However, this method results in a larger stencil for the viscous terms compared to the finite element discretization and could be more diffusive.

\section{Finite element discretizations}

Finite element method (FEM) merits a separate discussion since its evolution in CFD has taken place in parallel with finite volume schemes. The rigorous treatment adopted in FEM has important implications for global error estimation and grid adaptation. Many researchers have shown the equivalence between finite volume and finite element approaches when piecewise-linear approximations are employed for the solution vector and the fluxes $[147,72,11,150]$.

The most powerful method in finite elements is the Galerkin method. Here, the solution is first expanded in a set of basis functions and the residual is made orthogonal to a set of test functions. When the basis and the test functions are the same, the method is termed a Galerkin method; otherwise it is called a Petrov-Galerkin method. The standard Galerkin method leads to a centered scheme and is unconditionally unstable for hyperbolic problems when combined with forward Euler discretization in time. Therefore, artificial viscosity has to be added in some form to stabilize the procedure.

In contrast with the finite volume methods, finite element practitioners have always preferred cellvertex schemes since the global function is usually expressed as a summation of trial functions multiplied by the values at the vertices. These trial functions are typically assumed to be 1 at a vertex and zero at all other vertices. Bristeau et al. [28] computed many low Reynolds number compressible flows using a finite element method. Angrand and Dervieux [8] investigated several first and second order accurate explicit schemes. They added Lapidus-type artificial viscosity to a Galerkin method. Donea [41] derived the important Taylor-Galerkin family of schemes for the linear advection equation. Adopting an FEM approach, he showed how a Galerkin scheme (a centered scheme) could be stabilized by using a Taylorseries expansion for the time derivative $\frac{\partial u}{\partial t}$, similar to the procedure used to derive the Lax-Wendroff scheme. He demonstrated that the resulting schemes had good phase error and dissipation properties and that they could be easily extended to multi-dimensions. Löhner et al. [96] developed and tested a two-step Taylor-Galerkin finite element method for the solution of the Euler equations. Since the use of artificial viscosity spread the discontinuities over several cells, they also employed an adaptive grid algorithm using local refinement.

While there are finite difference/volume equivalents to most of the finite element techniques described in the previous paragraph, a very important class of schemes has been derived in the finite element community that has no obvious counterpart in the finite difference/volume approach. Included in this class are the Streamwise Upwind Petrov Galerkin (SUPG) or the Streamwise Diffusion (SD), and the Galerkin Least Squares methods. SUPG, devised originally by Hughes and Brooks [63] for the steady scalar advection-diffusion equation, and Galerkin least squares [64] methods have subsequently been extended to deal with the compressible and incompressible Navier-Stokes equations [62]. To the Galerkin discretization, they add terms proportional to the residual (unsteady residual for time-dependent problems) to introduce dissipation. Unlike conventional artificial viscosity, this form of artificial viscosity does not compromise the order of accuracy of the scheme since the exact solution yields zero dissipation. 
The global error in $L_{2}$ norm can been shown to vary as $h^{p+1 / 2}$ for the advection-dominated case and as $h^{p+1}$ for the diffusion-dominated case, where $p$ is the degree of piecewise-polynomial basis function [62]. Another method that has been extensively analyzed is the discontinuous Galerkin method [77, 33, 22]. Johnson et al. [78] have analyzed the SUPG scheme and have extended it to deal with time-dependent problems by adopting a discontinuous Galerkin procedure in time. A review and analysis of these and other finite element methods may be found in the text by Johnson [76]. All these methods are linear and are not suitable for capturing discontinuities. Hughes and Mallet [65] have proposed a shock-capturing operator that locally reduces the order of accuracy near discontinuities. Three-dimensional viscous flow over the canopy of a Hermes computed by Chalot et al. [32] is presented in Figures 3(a-b). The flow conditions corresponding to reentry are altitude of $60 \mathrm{~km}, M_{\infty}=20$, angle of attack $\alpha=30^{\circ}$ and $\operatorname{Re} / \mathrm{m}$ of 120,890 . The laminar solution was computed using equilibrium real gas hypothesis by the SUPG finite element method by an iterative implicit method that employs a block-diagonal preconditioned GMRES procedure. The mesh contains nearly 1 million tetrahedra. Figure 3a displays the surface grid and Figure $3 \mathrm{~b}$ depicts the skin friction lines, illustrating the complex flow structure.

\section{Turbulence modeling}

In order to compute practical flows at large Reynolds numbers, turbulence has to be modeled. Some of the most popular turbulence models in use in structured grids such as the Baldwin-Lomax [10] become quite difficult to implement on unstructured grids because of their nonlocal nature, although this has been done $[147,104]$. The trend is away from algebraic models and towards simple one and two equation field models. $k-\epsilon$ turbulence models have been used with unstructured grids either by integrating the model to the wall [168] or by using a two-layer model $[116,32]$, where the two equation $k-\epsilon$ model is replaced by a one equation model near the wall region. Marcum and Agarwal [100] have implemented two versions of $k-\epsilon$ models in an unstructured grid code to compute turbulent flows over an ONERA M6 wing. They tested a low Reynolds number model that is integrated to the wall and a high Reynolds number model that is combined with wall-functions. Two fairly new one-equation models have become very popular, particularly for unstructured grid applications. These are the Baldwin-Barth [9] and the Spalart-Allmaras [159] models. The Baldwin-Barth model is derived from a simplified form of the $k-\epsilon$ turbulence model. The Spalart-Allmaras model, on the other hand, is derived "from scratch" through dimensional analysis and intuition. Both the models have been tested extensively and are being used routinely. The cell-vertex finite volume scheme with a Galerkin procedure for computing the viscous terms has been used to compute viscous flows about high-lift configurations in conjunction with these turbulence models $[6,168]$. In Figure 4 , the surface pressure distributions and velocity profiles at three stations on the flap are displayed. The results are taken from the paper of Anderson and Bonhaus [6]. They use an implicit cell-vertex MUSCL scheme with the Spalart-Allmaras turbulence model, where the linear system at each time step is solved by a Gauss Seidel relaxation method. The results are compared with experimental data at two Reynolds numbers $5 \times 10^{6}$ and $9 \times 10^{6}$ with $M_{\infty}=0.2$ and angle of attack, $\alpha=16^{\circ}$. The surface pressure profiles show excellent agreement with the experimental data, while the velocity profiles show good agreement except for the wake regions far downstream, where the grid resolution is inadequate. These and other quantitative comparisons with experimental data [168] indicate the level of maturity of unstructured grid technology.

\section{Time Discretization and Solution Strategies}

\subsection{Steady-state solution techniques}

After discretizing Eqn. (1) in space, the following system of coupled ordinary differential equations is obtained:

$$
\frac{d(V M W)}{d t}+R(W)=0
$$


Here $W$ is the vector of unknowns over the mesh points for a vertex-based formulation and over the cells for a cell-based formulation. $V$ is the volume of the polyhedral control volume associated with the vertex/cell. In the case of a cell-vertex scheme storing pointwise quantities at the vertices, $M$ is the mass matrix which represents the relationship between the average value in a control volume and the values at the vertices (the vertex representing the control volume and its nearest neighbors). It is only a function of the mesh and hence, a constant matrix for a static mesh. If a steady state solution is sought, time-accuracy is not an issue and $M$ can be replaced by the identity matrix. This technique known as mass-lumping yields the following system of ordinary differential equations for the vector of unknowns $W$ :

$$
V \frac{d W}{d t}+R(W)=0
$$

Cell-centered schemes (up to second order accuracy) and schemes dealing strictly with cell-averages (to any order of accuracy) do not yield a mass matrix and thus lead to Eqn. ( 7 ) for steady and unsteady problems.

Eqn. 7 may be solved explicitly with linear multi-step methods of the forms described in [67, 170]. The coefficients for these Runge-Kutta schemes are derived by considering a model problem and optimizing the coefficients to yield a large CFL number and good damping properties. Local time stepping, enthalpy damping (for Euler equations) and residual averaging are employed to accelerate convergence [74]. Even with this methodology, the convergence to steady state is usually unacceptably slow. Therefore, either multigrid methods or implicit schemes are required to accelerate the convergence.

\subsubsection{Multigrid methods}

The multigrid method has been demonstrated as an efficient means for obtaining steady-state solutions to the compressible Euler and Navier-Stokes equations on unstructured meshes in two and three dimensions. In this approach, convergence acceleration is achieved by time-stepping on successively coarser meshes. The principle behind this algorithm is that the errors associated with the high frequencies are damped by a carefully chosen smoother (e.g. a multi-stage Runge-Kutta scheme) while the errors associated with the low frequencies are damped on the coarser grids where these frequencies manifest themselves as high frequencies. In the case of structured grids, coarse grids are easily derived from a given fine grid by omitting alternate grid lines in each coordinate direction. In the case of unstructured grids, three different approaches can be adopted.

The first approach begins with a coarse mesh definition and generates finer grids by refinement $[131,35]$. The advantage is that the inter-grid operators become simple because of the nesting of grids. Another advantage is that this set-up can be utilized to advantage in an adaptive procedure, where the fine meshes are formed by adaptively refining the coarse meshes [35, 127]. The principal disadvantage of this approach is the dependence of the fine grid distribution on the coarse levels. The second approach uses non-nested unstructured grids either with a subset of fine grid points comprising the coarse grids or with completely independent coarse and fine meshes. This has been shown to be successful in both for inviscid and viscous flow computations $[105,101,129,24]$. Both the approaches outlined above share a common problem, that of generating coarse grid levels. For complex geometries, especially in three dimensions, generating coarse grids that faithfully represent the complex geometries can become a difficult proposition. The requirement to generate not just one grid but multiple grids that preserve the geometry places too much of a burden on a user. The third approach circumvents this problem by generating coarse grids through agglomeration or fusing of fine grid control volumes, resulting in polyhedral coarse grid control volumes. This method was developed in [87] for cell-vertex schemes and in [158] for cell-centered schemes. This method has been further refined to deal with inviscid and viscous flows past complex configurations in both two and three dimensions $[85,177,108,109]$. In Figures 5 (a-e) the results from using the agglomeration multigrid strategy are presented. Figure 5a depicts the surface grid employed to compute inviscid flow about a low-wing transport configuration [177]. The mesh contains 804,056 vertices and approximately 4.5 million tetrahedra. Figure $5 \mathrm{~b}$ displays the computed 
surface Mach contours for transonic inviscid flow using a seven-level agglomeration multigrid strategy. The freestream conditions for this case are $M_{\infty}=0.77$ and $1.116^{\circ}$ incidence. Figure $5 \mathrm{c}$ shows the surface mesh employed for computing turbulent viscous flow over a partial span-flap wing experimental configuration [109]. The fine grid contains nearly 2.3 million vertices and nearly 13.6 million tetrahedra. The freestream conditions for this case are $M_{\infty}=0.2$, incidence of $10^{\circ}$, and Reynolds number of 2 million, corresponding to an approach condition. The solution obtained with a six-level agglomeration multigrid strategy is shown qualitatively in Figure $5 \mathrm{~d}$ in the form of Mach contours on the wind-tunnel wall and density contours on the wing surface. Figure 5e shows the convergence histories for these two cases. The deterioration in convergence of the Navier-Stokes case is mainly due to the use of stretched meshes and indicates that the efficiency of Navier-Stokes solvers is far from satisfactory.

\subsubsection{Implicit schemes}

Implicit schemes for the compressible Euler and Navier-Stokes equations have been developed in order to accelerate the convergence to steady state. If the time derivative in Eqn. (7) is replaced by:

$$
\frac{d W}{d t}=\frac{W^{n+1}-W^{n}}{\Delta t}
$$

an explicit scheme is obtained by evaluating $R(W)$ at time level $n$. An implicit scheme is obtained by evaluating $R(W)$ at level $n+1$. In the latter case, linearizing $R$ about time level $n$, we obtain

$$
\begin{gathered}
\left(\frac{V}{\Delta t}+\frac{\partial R}{\partial W}\right) \Delta W_{i}=-R_{i}, \\
\Delta W_{i}=\left(W^{n+1}-W^{n}\right)_{i} .
\end{gathered}
$$

Eqn. (9) represents a large nonsymmetric linear system of equations for the updates of the vector of unknowns and needs to be solved at each time step. As $\Delta t$ tends to infinity, the method reduces to Newton's method. Direct solvers have been used solve Eqn. (9) yielding quadratic convergence, but these entail prohibitive costs and memory requirements and are impractical for three dimensional applications $[176,157]$. Typically, due to storage considerations, only a lower order representation of the left-hand side is employed and the system is solved by iterative means. As a result of this approximation Eqn. (9) can never approach Newton's method (with its associated quadratic convergence property) due to the mismatch of the right and left hand side operators.

Thareja et al. [163] and Hassan et al. [58] have utilized point-implicit iterative procedures. Fezoui [45], Batina [17], Anderson et al. [5, 7] and Slack et al. [157] have used a Gauss-Seidel relaxation technique. It is also possible to use more sophisticated techniques for the solution of the linear system, such as GMRES [149] and QMR [46]. Preconditioning of the matrices is critical to achieving good convergence with these methods. Some of the typical preconditioners are block-diagonal, symmetric successive over-relaxation (SSOR) and Incomplete LU factorization (ILU) preconditioners [111]. GMRES with diagonal preconditioning has been used by Shakib et al. [151] to solve the linear systems arising out of a finite element discretization of the Euler equations. Slack et al. [157] and Whitaker [184] have also used GMRES with diagonal preconditioning in two and three-dimensional applications. Slack et al. [157] have observed when solving the two-dimensional Euler equations that the preconditioned iterative methods perform better than the other methods as the number of elements in the mesh increases. Venkatakrishnan and Mavriplis [178] tested a family of implicit schemes for solving the two-dimensional compressible Navier-Stokes equations on unstructured meshes. They concluded that GMRES with ILU preconditioning (GMRES/ILU) was superior to other implicit schemes over a range of problems sizes and flow conditions and was competitive in terms of CPU time with multigrid methods. Luo et al. [99] used GMRES/ILU to compute two- and three-dimensional flows. A drawback of all the methods based on linear algebra is that the memory requirements become severe which seriously limits the sizes of the problems that can be solved, especially in three dimensions. It is possible to implement GMRES 
in a matrix-free form [29] where the matrix-vector product is replaced by a finite difference expression involving residual evaluations. Another advantage of the matrix-free approach is that the higher order discretization can be employed for the left-hand side and the matrix-vector product [30]. The drawback of this approach is that powerful preconditioners like SSOR and ILU cannot be used since they require the matrix to be explicitly available. A compromise is to settle for a matrix-free form of GMRES with diagonal preconditioning as done in [75].

Another implicit method that has been investigated is based on the use of linelets [59]. The key idea is to cover the domain with a set of lines and the scheme is made implicit along these lines. Thus tridiagonal systems need to be solved which can be accomplished efficiently. A drawback of this method is that the convergence is sensitive to the orientation of the linelets with respect to the direction of strongest coupling.

\subsection{Unsteady Problems}

While the solution techniques for computing steady flows have evolved to a high degree, those for dealing with unsteady flows have lagged behind. This is partly due to fact that prediction of aerodynamic properties in steady transonic flow (cruise conditions) has been the driving force, with the unsteady effects being important only in extreme conditions, such as flutter and stall. It is anticipated, however, that unsteady time-accurate simulations will provide the next great challenge for CFD.

Recall that after discretization in space, a system of coupled ODE's results (Eqn (6)). If we assume that the system is decoupled i.e. $M \equiv I$, the simplest way to solve the system of ODE's is to use an explicit scheme. Low-storage Runge-Kutta methods $[67,170]$ and various predictor-corrector schemes fall under the category of explicit schemes. These schemes typically require only simple updates. They place restrictions on time step due to the CFL condition, which become severe in the case of semidiscrete schemes satisfying monotonicity principles. However, explicit schemes may be the schemes of choice for certain unsteady applications when the time scales of interest are small or more precisely, that they are comparable to the spatial scales. The grid should be clustered only in regions of interest; otherwise, the size of the explicit time step could be unnecessarily small. The situation can be improved by the use of time-step sequencing $[83,138]$, where different cells take varying number of local time steps to get to a particular time level.

When the mass matrix is present, even the explicit schemes require a matrix inversion. Often, either the mass-matrix is lumped for convenience or at best a few Jacobi iterations are carried out $[124,36]$. Neither approach is entirely satisfactory, especially if large time steps, permitted by multi-stage explicit schemes and implicit schemes, are used. Donea [41] realized that the presence of the mass matrix in his formulations will make it unattractive and proposed a simple two-pass procedure. While he proved that the formal order of accuracy remained the same, he also showed that the dissipation and dispersion properties were somewhat compromised. Recently, it has been observed [179] that the mass matrix can be lumped without adverse consequences if the cell-vertex scheme possesses only second or lower order accuracy.

When an implicit scheme is used to solve for unsteady flows, the linear system Eqn.(9) is modified by replacing the diagonal matrix $V$ by the matrix $M V$ where $M$ is the mass matrix and $V$ is the volume of the cell. However, it is not enough to solve this linear system. One has to drive the unsteady residual to zero or at least to truncation error. This is usually done by employing inner iterations $[139,136]$. It is the role of these inner iterations to eliminate errors due to the factorization (if any is carried out), linearization, and errors arising from employing a lower order approximation on the implicit side. The number of inner iterations required may be large depending on the flow situation and the size of the time step employed.

Brennis and Eberle [2]] and Jameson [68] have advocated a different approach for deriving an efficient implicit scheme for unsteady flows.The idea is to define an unsteady residual, following a backward difference approximation to the time derivative and then use either a relaxation strategy or a multigrid technique to drive the unsteady residual to zero. The significant advantage of this approach 
when multigrid is used to solve the nonlinear problem is that it incurs no storage overheads associated with traditional implicit schemes, and is particularly attractive for unstructured grid computations in three dimensions. It allows the time step to be determined solely based on flow physics. This method has been used to compute two- and three-dimensional flows over airfoils and wings $[68,112,4]$ using structured grids. Vassberg [172] has applied this method to compute flow solutions over oscillating airfoils using unstructured grids. This approach has also been used in [179] in conjunction with the agglomeration multigrid strategy to solve two-dimensional unsteady flows where the inversion of the mass matrix is accomplished indirectly during the multigrid procedure.

\section{Adaptive grids}

Solution adapted grids are increasingly being used to compute complex unsteady and steady flows. There are three distinct ways the grid can be adapted to the solution. These are r-refinement, hrefinement and p-refinement. In r-refinement, the nodes are simply redistributed so that regions of importance are better resolved. In h-refinement or mesh-enrichment, the cells are locally subdivided or merged or in some instances, a complete remeshing is done to reduce the grid spacing in regions of interest. In p-refinement, the degree of the basis function is adjusted locally to match the variation in solution. For a survey on adaptive mesh refinement techniques, the reader is referred to the review article by Powell et al. [135].

$\mathrm{R}$-refinement is probably the simplest in concept, but is burdened with practical difficulties in multidimensions especially when dealing with highly stretched grids. For a survey of mesh point movement methods, see the review article by Hawken et al. [60]. The difficulties include skewness, crossing of lines, arbitrarily small cell volumes etc. Some progress has been made in dealing with these issues at least for inviscid flows, where the cell-aspect ratios are not too severe [122]. The advantage of $\mathrm{r}$ refinement is that if a valid grid results from it, all that is required is the interpolation of variables from the old to the new grid. This could be done in a conservative manner if desired. A way to avoid the interpolation, which introduces errors that could accumulate, is to introduce the grid movement terms in the governing equations Eqn. (1). These terms need to be discretized carefully so that freestream is preserved. The Geometric Conservation Law (GCL) $[165,186]$ formalizes this procedure. Recently, r-refinement has been used to great advantage with Roe's upwind scheme [143] to obtain "fitted" shock resolution by Paraschivoiu et al. [123], Parpia and Parikh [126], and van Rosendale [171] by aligning edges of the triangulation with discontinuities. The "fitting" is done in a shock-capturing framework by utilizing that property of Roe's scheme which allows isolated discontinuities aligned with the mesh to be captured exactly.

H-refinement is by far the most popular means of adaptation in compressible flows. This is especially true for inviscid flows dominated by interactions of shock waves where p-refinement techniques are of limited value. In the Adaptive Mesh Refinement (AMR) approach, h-refinement is employed with bodyfitted structured grids to adapt to the flow solution [20,138]. Instead of body-fitted grids, Cartesian meshes can be used to adapt to complex geometries in addition to adapting to flow features [21, 37, 137$]$. Since some of the approaches, e.g. [37, 152] do not make use of any structure and employ indirect addressing, these methods may be classified as unstructured. The AMR framework has been used to compute many complex inviscid unsteady flows that exhibit disparate spatial and temporal scales. Unstructured grids, composed of triangles or tetrahedra, are also particularly suited for h-refinement. In the case of both AMR and unstructured grids, the regions of interest are first identified either through a combination of heuristic criteria such as density gradients (undivided) or through estimation of the truncation error. In the case of AMR, typically cells are subdivided in some fixed ratio, and these are sometimes grouped into logically rectangular patches. In the case of unstructured grids, the regridding procedure is more involved. If a Delaunay triangulation is used as the initial grid, one can use Watson's incremental algorithm [183] to obtain a new Delaunay triangulation. Other procedures such as edgeswapping may also be used to restore the Delaunay triangulation. In the case of other triangulations, 
cells are subdivided and smoothed using a few passes of a Laplacian-type smoother. Peraire et al. [130] advocate the regeneration of the grid by the advancing front method for steady state applications after identifying regions of interest by error estimation. In the case of nodal schemes in three dimensions, when edges are tagged and the cells are to be refined or derefined based on these tagged edges, the possibilities are numerous [79]. It helps to use some rules to limit the cases [35]. Several investigators have employed multigrid procedure for steady state computations using the grids generated by solution adaptation $[102,12 \%, 35,26]$. Figure 6 a shows the initial surface grid for an isolated engine inlet. The initial grid contained 3536 nodes. After three adaptive refinement cycles, the final mesh shown in Figure $6 \mathrm{~b}$ was obtained. The final mesh contained 61,002 nodes. This computation done by Connell and Holmes [35] utilized the adaptive multigrid procedure. When adapting grids to flow features, a problem that may occur is that certain regions are overly resolved at the expense of other regions, resulting in seemingly sharp but incorrect solutions. Warren et al. [182] have proposed and tested a simple modification to the error indicators that alleviates this problem.

In the case of transient problems, adaptation is performed much more frequently and therefore the regridding process is required to be efficient. While this is easily accomplished efficiently by subdivision of cells in the AMR framework, the problem is more involved in the case of unstructured grids [94, 141]. The problem of flow past bodies in relative motion has also been addressed in the literature [93, 167, $57,82,31]$. Typically, mesh point movement and efficient mesh restructuring are employed to obtain valid, good-quality grids about the moving bodies. Figures $7(\mathrm{a}-\mathrm{c})$ show the results from the simulation of store separation from F-117. The freestream conditions are $M_{\infty}=0.84$ and incidence of $2.73^{\circ}$. The projectiles are forcibly ejected at different velocities. This multi-body problem was computed by Baum et al. using the Arbitrary Euler Lagrangian adaptive unstructured methodology developed in [18]. Because of symmetry, the motion of only two of the projectiles is simulated. Figures $7 \mathrm{a}$ and $7 \mathrm{~b}$ show the Mach contours from two different perspectives and Figure $7 \mathrm{c}$ displays the pressure contours at a particular instant of motion. The complex shock patterns emanating from the projectiles and their signatures on the plane of symmetry may be observed in Figure $7 \mathrm{c}$.

\section{Higher order methods}

The use of methods possessing higher than second order accuracy for solving the compressible NavierStokes equations on unstructured grids is not yet commonplace and is a topic of active research. The finite element method achieves higher order accuracy by the use of higher order elements. Bey and Oden [22] have used a discontinuous Galerkin method to obtain up to 4th order accuracy for smooth flows using structured adaptive grids. In the finite volume community, Barth and Fredrickson [14] derived general conditions for a scheme to be higher order accurate that involve the reconstruction of variables satisfying the properties of conservation of mean, $k$-exactness and compact support. $k$ exactness refers to the property of being able to reconstruct exactly polynomials of degree $\leq k$. The key idea is to extend the support of the scheme to enable the coefficients in the polynomial to be determined. They also proposed a minimum-energy reconstruction that used a larger support and utilized a least-squares method to evaluate the coefficients. By combining this reconstruction procedure with higher order quadrature, they demonstrated the effectiveness of both $h$ - and $p$-refinements in reducing the errors in smooth flows. More recently, Chakravarthy et al. [31] have proposed a similar approach to achieving higher order accuracy. Equivalent to requiring $k$-exactness, they propose that the mean be conserved in a neighborhood of the point in question. Mitchell [115] has proposed a quasiquadratic reconstruction procedure that appears to yield better results than those obtained with linear reconstruction. Harten and Chakravarthy [55] have proposed a framework for applying Essentially Nonoscillatory (ENO) schemes [56] on unstructured meshes. They present two techniques for adapting the stencils during the reconstruction procedure, which also give preference to a centered stencil in smooth regions. The first technique considers several candidatestencils and picks the one that represents the function the smoothest. The second technique is a hierarhical one that incrementally augments the 
stencil as derivatives are required during the reconstruction procedure. Abgrall [2] has also proposed a hierarchical ENO scheme and has tested it by computing a variety of inviscid compressible flows.

A problem with the reconstruction methods described above is that since the support grows with the order of the scheme, computational cost and boundary treatment become significant. Halt and Agarwal [53] have proposed and tested two methods for realizing higher order accuracy while keeping the support compact. These techniques are based on deriving equations for either the derivatives or the moments of the governing equations. They demonstrate up to 4th order accuracy with both these methods for smooth flows. They conclude that the method based on moments is more suitable for dealing with shocks and boundary conditions. Barth [13] has also proposed a different quadratic reconstruction procedure. Similar to a finite element procedure, additional degrees of freedom are placed at the mid-points of edges in a triangulation. The gradient and Hessian required for quadratic reconstruction are again obtained by a least-squares procedure. Figures $8(\mathrm{a}-\mathrm{d})$, provided by Barth [13], illustrate the results obtained with various discretizations for the circular advection problem:

$$
u_{t}+(y u)_{x}-(x u)_{y}=0 .
$$

Discontinuous initial data is specified along an interior cut line. The exact solution is a solid body rotation of the cut-line data. The triangular grid is displayed in Figure 8a. Solutions obtained with the finite volume scheme employing piecewise-constant, piecewise-linear and piecewise-quadratic reconstructions are depicted in Figures $8(\mathrm{~b}-\mathrm{d})$. The significant improvement with higher order reconstruction is apparent. Barth [13] also states that quadratic reconstruction is about 7 times as expensive as linear reconstruction; of this a factor of 4 arises from the introduction of grid points at the mid-points of the edges. This "h-refinement" contributes in part to the improvement realized with quadratic reconstruction.

\section{$9 \quad$ Hybrid discretizations}

While triangulations in two and three dimensions offer flexibility in adapting to complex geometries, it is not clear if they are needed near the wall regions. Steger [161] when introducing the "thinlayer" approximation in 1978 stated, "One does not generally have sufficient computer to resolve the viscous terms except in a thin-layer near the body". This statement holds true even today for practical aerodynamic computations. Thus highly stretched triangulations are required in the near-wall regions. They can be generated by employing a mapping procedure [10.3] where a Delaunay triangulation is performed in the mapped plane which is then mapped back to the physical space to yield the desired stretched triangulation. This method has also been used for adaptation in viscous flows [181]. Aftosmis et al. [3] have addressed the important issue of element shapes by examining the accuracy obtained on test problems by using various triangular element shapes and quadrilaterals. They conclude that using stretched triangulations near the body does not yield any improvement in solution accuracy over using quadrilaterals, and is therefore inefficient because of the extra edges in the triangulation. They recommend a method that removes unnecessary edges (diagonals) from boundary layer regions. Rather than adopt this two-step procedure, where the triangular grid is first generated followed by the elimination of unnecessary edges, the hybrid method eliminates the need for stretched triangulations all together. This method makes use of a structured or a semi-structured grid in the near-wall regions. In two dimensions, a structured body-fitted grid is used near the body whereas in three dimensions a prismatic grid is used since the surface grid is triangular. Nakahashi et al. [117, 118], Kallinderis et al. [80, 128], Connell and Braaten [34] have advocated this approach. The generation of prismatic elements requires careful marching away from the surface so that lines do not cross. Melton et al. [113] have used a prismatic grid with a background Cartesian mesh to compute three-dimensional flows. The structure afforded could be effectively used to tailor line-implicit algorithms, although this requires that the various elements be processed separately. Ideally, one data structure (edge-based) should be able to process all elements regardless of their shapes for an automatic procedure. Figure 9a shows the surface 
grid for an adapted hybrid prismatic-tetrahedral grid about a hemisphere. The initial grid contained 3000 triangular faces on the wall and 20 layers of prisms and 70,000 tetrahedra. The freestram conditions for supersonic laminar flow are $M_{\infty}=1.4$ and $R e=1000$. After the dual adaptation procedure, where the prisms are directionally refined with the normal resolution unaltered and the tetrahedra are divided into either 2,4 , or 8 tetrahedra, the final grid contains 7000 triangular faces and 20 layers of prisms and 225,000 tetrahedra. The signature of the directional refinement of the prisms may be observed on the hemispherical surface. Figure $9 \mathrm{~b}$ displays the surface Mach contours on the symmetry plane for this flow. These figures have been provided by Parthasarathy et al. [128]. The explicit solver also makes use of an adaptive multigrid procedure.

\section{Parallel computing}

Computational fluid dynamics as its name implies is inevitably linked to computing issues. Among these are processing power, memory technology, networking and accessibility. Ability to compute the solutions to problems in finite time always being the goal, CFD has benefited immensely from the revolution that has taken place in the last 15 years in these areas. Vector supercomputers have provided much of the computing power that has been harnessed to compute complex three-dimensional flows. It is anticipated that distributed-memory parallel computers will offer the next cost-effective leap forward in terms of computing power. Much research has been carried out in the area of unstructured grid computations on parallel computers. Some of the issues that arise are partitioning of the grid, message patterns, data structures and design of parallel algorithms. Partitioning of unstructured grids for parallel computing has been investigated by a number of researchers. The methods can be broadly classified into geometry-based $[23,114,44]$ and graph-based $[133,156]$ algorithms. It appears that the graph-based algorithms, in particular, the spectral bisection technique of Pothen et al. [133], while being computationally more expensive, yield much better partitions. Unstructured grid flow solvers have been implemented on various machines $[180,107,75,43,140]$. These studies have shown that good performance may be obtained by paying careful attention to the issues listed above. Regarding the flow solver, explicit schemes and point Jacobi implicit schemes possess almost complete parallelism, except for communication at the inter-processor boundaries. Multigrid methods appear to have adequate parallelism for coarse-grained parallel computers; impressive performances have been reported by Mavriplis et al. [107]. Johan et al [75], Ramamurthi et al. [140] and Venkatakrishnan [175] have shown that implicit schemes can be carefully designed to yield good performance when solving unstructured grid problems on parallel computers. While a matrix-free GMRES with nodal block preconditioner that has almost complete parallelism except for the communication at the inter-processor boundaries is used in [75], methods that are implicit within processors but explicit across processors are used in $[140,175]$. Thus, as the number of processors increases, the latter methods exhibit a degradation in convergence. In [175] it is demonstrated that convergence rate of such a method can be improved by using in addition a global coarse grid approximation, where each processor is represented by one grid point. In general, it appears that the best parallel algorithm is also the best serial one, with some possible loss in convergence in the case of implicit schemes in a multi-processor environment.

\section{Conclusions}

In this paper, unstructured grid flow solvers for the compressible Navier-Stokes equations have been surveyed. Significant progress has been made in the areas of spatial and temporal discretization, adaptive and parallel algorithms. Newly developed field equation turbulence models seem to mesh nicely with the unstructured grid framework. Based on these developments, it is safe to say that unstructured grid technology is almost on par with structured grid technology, although encumbered with additional memory and computational costs. This overhead has to be balanced with the ability to compute flows over complex geometries and the ease of adaptation. 
The areas that require further research are thus the same as in structured grids. These include better and faster implicit/multigrid flow solvers that are insensitive to cell aspect ratios and grid stretching. improved higher order discretization techniques, better a priori or a posteriori error estimates and parallel algorithms. The status of unsteady flow solvers is far from satisfactory either for structured or unstructured grids. The use of hybrid meshes needs to be explored further to utilize data structures that can handle any type of tessellation. As unsteady flows and aeroacoustics are emerging as areas of interest, nondissipative schemes that also minimize dispersion have to be designed to be applicable to unstructured grids, which are nonuniform in general. In this respect as well as in the areas of error estimation and higher order schemes, there should be more interaction between finite volume and finite element communities.

Acknowledgements. The author would like to thank the following researchers for their contributions: W. K. Anderson, T. J. Barth, J. D. Baum, B. Stoufflet, S. D. Connell, N. T. Frink, A. Jameson, Y. Kallinderis, R. Löhner, L. Martinelli, and D. J. Mavriplis. In addition, the comments of W. K. Anderson, D. J. Mavriplis, J. J. Quirk, and D. Sidilkover on the earlier drafts are greatly appreciated.

\section{References}

[1] Special course on unstructured grid methods for advection dominated flows, vol. AGARD Report 787, AGARD, 1992.

[2] R. ABgrald, On Essentially Non-oscillatory schemes on unstructured meshes: Analysis and implementation, J. Comp. Plys., 114 (1994), pp. 45-58.

[3] M. Aftosmis, D. Gaitonde, And T. S. Tavares, On the accuracy, stability and monotonicity of various reconstruction algorithms for unstructured meshes. AIAA Paper 94-0415, Jan. 1994.

[4] J. Alonso AND A. JAmeson, Fully-implicit time-marching aeroelastic solution. AIAA Paper 94-0056, Jan. 1994.

[5] W. K. Anderson, A grid generation and flow solution method for the Euler equations on unstructured grids, J. Comp. Phys., 110 (1994), pp. 23-38.

[6] W. K. Anderson And D. L. Bonhaus, Navier-Stokes computations and experimental comparisons for multielement airfoil configurations. AIAA Paper 93-0645, Jan. 1993.

[7] - An implicit upwind algorithm for computing turbulent flows on unstructured grids, Computers Fluids, 23 (1994), pp. 1-21.

[8] F. Angrand and A. Dervieux, Some explicit triangular finite element schemes for the Euler equations, Int. J. for Numer. Meth. in Fluids, 4 (1984), pp. 749-764.

[9] B. S. BALDWin AND T. J. BARTh, A one-equation turbulence transport model for high Reynolds wall-bounded flows. AIAA Paper 91-0610, Jan. 1991.

[10] B. S. BALDWIN AND H. J. Lomax, Thin layer approximation and algebraic model for separated turbulent flows. AIAA Paper 78-257, Jan. 1978.

[11] T. J. BARTH, Numerical aspects of computing viscous high Reynolds number flows on unstructured meshes. AIAA Paper 91-0721, Jan. 1991.

[12] — A three-dimensional upwind Euler solver for unstructured meshes. AIAA Paper 91-1548CP, July 1991. 
[13] - Recent developments in high order k-exact reconstruction on unstructured meshes. AIAA Paper 93-0668, Jan. 1993.

[14] T. J. BARTH AND P. O. FREDRICKSON, Higher order solution of the Euler equations on unstructured grids using quadratic reconstruction. AIAA Paper 90-0013, Jan. 1990.

[15] T. J. BARTH AND D. C. JESPERSEN, The design and application of upwind schemes on unstructured meshes. AIAA Paper 89-0366, Jan. 1989.

[16] J. T. BATINA, Implicit flux-split Euler scheme for unsteady aerodynamic analysis involving unstructured dynamic meshes, AIAA J., 29 (1991), pp. 1836-1843.

$[17]+$ Implicit upwind solution algorithms for thre€-dimensional unstructured meshes, AIAA J., 31 (1993). pp. 801-805.

[18] J. D. BAUM, H. LUO, AND R. LÖHNER, A new ALE adaptive unstructured methodology for the simulation of moving bodies. AIAA Paper 94-0414, Jan. 1994.

[19] J. A. Benek, P. G. Buning, and J. L. Steger, A 3-d chimera grid embedding technique. AIAA Paper 85-1523, July 1985.

[20] M. J. Berger AND P. Colella, Local adaptive mesh refinement for shock hydrodynamics, .1. Comp. Phys., 82 (1989), pp. $67-84$.

[21] M. J. Berger and R. J. Leveque, An adaptive Cartesian mesh algorithm for the Euler equations in arbitrary geometries. AIAA Paper 89-1930CP, June 1989.

[22] K. S. BEY AND J. T. OdEN, A Runge-Kutta local projection P1-discontinuous Galerkin finite element method for high speed flows. AIAA Paper 91-1575CP, July 1991.

[23] S. Bokhari, T. W. Crockett, and D. M. Nichol, Parametric nested dissection. ICASE Report 94-39, 1994.

[24] D. L. BonHAUS, An upuind multigrid method for solving viscous flows on unstructured triangular meshes, Master's thesis, George Washington Univ., 1993.

[25] J. P. BORIS AND D. L. Book, Flux corrected transport, 1 SHASTA, a fluid transport algorithm that works, J. Comp. Phys., 11 (1973), pp. 38-69.

[26] M. E. Bratien and S. D. Connell, A 3-d unstructured adaptive multigrid scheme for the Navier-Stokes equations, Tech. Rep. 94CRD146, GE Research and Development Center, Aug. 1994.

[27] A. Brennis AND A. EBerLe, Application of an implicit relaxation method solving the Euler equations for time-accurate unsteady problems, Transactions of ASME - J. Fluids Engrg., 112 (1990), pp. 510-520.

[28] M. O. Bristeau, R. Glowinski, B. Dimoyat, J. Periaux, P. Perrier, and O. Prionneau, Finite element methods for the compressible Navier-Stokes equations. AIAA Paper 83-1890, July 1983.

[29] P. N. BROWN AND Y. SAAD, Hybrid krylov methods for nonlinear systems of equations, SIAM J. Sci. Stat. Comput., 11 (1990), pp. 450-481.

[30] X. C. CAI, W. D. Gropp, D. E. Keyes, And M. D. Tidriri, Newton-hrylov-Schwarz methods in CFD, in Proceedings of the International Workshop on Numerical Methods for the NavierStokes Equations, vol. 47 of Notes in Numerical Fluid Mechanics, Braunschweig/Wiesbaden, 1994, Vieweg Verlag. 
[31] S. Chakravarthy, K. Y. Szema, S. Ramakrishnan, R. Burman', and R. Schultz, Inviscid CFD for store separation using unified boundary conditions. AIAA Paper 93-3404, Aug. 1993.

[32] F. Chalot, M. Mallet, and M. Ravachol, A comprehensive finite element Navier-Stokes solver for low and high-speed aircraft design. AIAA Paper 94-0814, Jan. 1994.

[33] B. Cockburn, S. Hou, and C. Shu, TVB Runge-Kutta local projection discontinuous Galerkin finite element method for conservation laws IV: the multidimensional case, Math. Comp., 54 (1990), pp. 545-581.

[34] S. D. Connell and M. E. Braten, Semi-structured mesh generation for 3d Navier-Stokes calculations, Tech. Rep. 94CRD154, GE Research and Development Center, Aug. 1994.

[35] S. D. Connell and D. G. Holmes, A $3 d$ unstructured adaptive multigrid scheme for the Euler equations, AIAA J., 32 (1994), pp. 1626-1632.

[36] G. A. Davis And O. O. Bendiksen, Unsteady transonic two-dimensional Euler solutions using finite elements, AIAA J., 31 (1993), pp. 1051-1059.

[37] D. De Zeetw and K. G. Powell, An adaptively refined cartesian mesh solver for the Euler equations, J. Comp. Phys., 104 (1993), pp. 56-69.

[38] H. Deconinck, C. Hirsch, and J. Peuteman, Characteristic decomposition methods for the multidimensional Euler equations, Lecture Notes in Physics, 264 (1986).

[39] H. Deconinck, H. Paillere, R. Struijs, and P. L. Roe, Multidimensional upwind schemes based on fluctuation-splitting of conservation laws, Comp. Mechanics, 11 (1993), pp. 323-340.

[40] J. A. Desideri AND A. Dervieux, Compressible flow solvers using unstructured grids, VhI Lecture Series, 1988-05 (1988), pp. 1-115.

[41] J. DoneA, A Taylor-Galerkin method for convective transport problems, Int. J. for Numer. Meth. in Engrg., 20 (1984), pp. 101-119.

[42] L. J. Durlofsky, B. Engquist, And S. Osher, Triangle-based adaptive stencils for the solution of hyperbolic conservation laws, J. Comp. Phys., 98 (1992), pp. 64-73.

[43] C. Farhat, L. Fezoul, and S. Lanteri, Two-dimensional viscous flow computation on the Connection Machine: Unstructured meshes, upwind schemes and parallel computation, Comput. Methods Appl. Mech. Engrg, 102 (1993), pp. 61-88.

[44] C. FARHAT AND M. Lesoinne, Automatic partitioning of unstructured meshes for the parallel solution of problems in computational mechanics, Intl. J. for Numer. Meth. in Engrg., 36 (1993), pp. $745-764$.

[45] L. Fezoui And B. Storfflet, A class of implicit upwind schemes for Euler simulations with unstructured meshes, J. Comp. Phys., 84 (1989), pp. 174-206.

[46] R. W. Freund, G. H. Golub, and N. M. Nachtigal, Iterative solutions of linear systems, Acta Numerica, 1 (1992), pp. 57-100.

[47] N. T. FRINk, Upwind scheme for solving the Euler equations on unstructured tetrahedral meshes, AIAA J., 30 (1992), pp. 70-77.

[48] - Recent progress toward a three-dimensional unstructured Navier-Stokes flow solver. AIAA Paper 94-0061, Jan. 1994. 
[49] N. T. Frink, P. Parikh, and S. Pirzadeh, Aerodynamic analysis of complex configurations using unstructured grids. AIAA Paper 91-3292, Sept. 1991.

[50] P. L. Garner, P. T. Meredith, and R. C. Stoner, Areas for future CFD development as illustrated by transport aircraft applications. AIAA Paper 91-1527CP, July 1991.

[51] A. G. Godfrey, C. R. Mitchell, and R. W. Walters, Practical aspects of spatially high accurate methods. AIAA Paper 92-0054, Jan. 1992.

[52] S. K. Godvnov, A difference method for the numerical calculation of discontinuous solutions of the hydrodynamic equations, Mat.-Sb., 47 (1959).

[53] D. W. HALT AND R. K. AGARwAL, Compact higher order characteristic-based Euler solver for unstructured grids, AIAA J., 30 (1992), pp. 1993-1999.

[54] A. HARTEN, On a class of high-resolution total-variation-stable finite difference schemes, SIAM J. Numer. Anal., 21 (1984), pp. 1-23.

[55] A. HARTEN AND S. Chakravarthy, Multi-dimensional ENO schemes for genetal geometries. ICASE Report No. 91-76, 1991; submitted to J. Comp. Phys.

[56] A. Harten, B. Engquist, S. Osher, and S. Chakravarthy, Uniformly high order accurate Essentially Non-Oscillatory schemes III, J. Comp. Phys., 71 (1987), pp. 231-303.

[57] J. E. Hase, D. A. Anderson, And I. PARPiA, A Delaunay triangulation method and Euler solver for bodies in relative motion. AIAA Paper 91-1590CP, July 1991.

[58] O. Hassan, K. Morgan, and J. Peraire, An adaptive implicit/explicit finite element scheme for compressible high speed flows. AIAA Paper 89-0363, Jan. 1989.

[59] —. An implicit finite element method for high speed flows. AIAA Paper 90-0402, Jan. 1990.

[60] D. F. HAWKEN, Review of some adaptive node-movement techniques in finite-element and finitedifference solutions of partial differential equations, J. Comp. Phys., 95 (1991), pp. 254-302.

[61] D. G. Holmes And S. D. Connell, Solution of the 2D Navier-Stokes equations on unstructured adaptive grids. AIAA Paper 89-1392, June 1989.

[62] T. J. R. HuGHES, Recent progress in the development and understanding of SUPG methods with special reference to the compressible Euler and Navier-Stokes equations, Intl. J. for Numer. Meth. in Fluids, 7 (1987), pp. 1261-1275.

[63] T. J. R. Hughes AND A. Brooks, A multidimensional upwind scheme with no crosswind diffusion, in Finite element methods for convection dominated fows, New York, 1979, ASME.

[64] T. J. R. Hughes, L. P. Franca, ANd G. M. Hulbert, A new finite element formulation for computational fluid dynamics: VIII The Galerkin/least-squares method for advective-diffusive systems, Comput. Methods Appl. Mech. Engrg, 73 (1989), pp. 173-189.

[65] T. J. R. Hughes AND M. MALlet, A new finite element formulation for CFD: IV A discontinuity-capturing operator for multi-dimensional advective-diffusive systems, Comput. Methods Appl. Mech. Engrg, 58 (1986), pp. 329-336.

[66] D. HumMEL, On the vortex formation over a slender wing at large angles of incidence. AGARD CP-247, Paper No. 15, Feb. 1983. 
[67] A. JAMESON, Solution of the Euler equations by a multigrid method, Applied Mathematics and Computation, 13 (1983), pp. 327-356.

[68] - Time-dependent calculations using multigrid, with applications to unsteady flows past airfoils and wings. AIAA Paper 91-1596, July 1991.

[69] _. Analysis and design of numerical schemes for gas dynamics 1. Artificial diffusion, upuind biasing, limiters and their effect on accuracy and multigrid convergence. Submitted to Intl. J. Comp. Fluid Dynamics, 1994.

[70] - Analysis and design of numerical schemes for gas dynamics 2. Artificial diffusion and discrete shock structure. Submitted to Intl. J. Comp. Fluid Dynamics, 1994.

[71] A. JAmeson AND T. J. BAKer, Improvements to the aircraft Euler method. AIAA Paper 87-0452, Jan. 1987.

[72] A. Jameson, T. J. Baker, and N. P. Weatherill, Calculation of inviscid transonic flow over a complete aircraft. AIAA Paper 86-0103, Jan. 1986.

[73] A. Jameson AND D. J. MaVRIPlis, Finite volume solution of the two-dimensional Euler equations on a regular triangular mesh, AIAA J., 24 (1986), pp. 611-618.

[74] A. Jameson, W. Schmidt, and E. Turkel, Numerical solution of the Euler equations by finite volume methods using Runge-Kutta time stepping schemes. AIAA Paper 81-1259, 1981.

[75] Z. Johan, T. J. R. Hughes, K. K. Mathur, And S. L. Johnsson, A data parallel finite element method for computational fluid dynamics on the Connection Machine System, Comput. Methods Appl. Mech. Engrg., 99 (1992), pp. 113-124.

[76] C. Johnson, Numerical solution of partial differential equations by the finite element method, Cambridge University Press, New York, USA, 1992.

[7i] C. Johnson, U. Nävert, and J. Pitkäranta, Finite element methods for linear hyperbolic problems, Comput. Methods Appl. Mech. Engrg, 45 (1984), pp. 285-312.

[78] C. Johnson, A. Szepessy, And P. Hansbo, On the convergence of shock-capturing streamlinediffusion finite element methods for hyperbolic conservation laws, Math. Comp., 54 (1990), pp. $10 \bar{\imath}-130$.

[79] Y. KALlinderis AND P. VIJAYAN, Adaptive refinement-coarsening scheme for three-dimension al unstructured meshes, AIAA J., 31 (1993), pp. 1440-1447.

[80] Y. KALLINDERIS AND S. WARD, Hybrid prismatic/tetrahedral grid generation for complex geometries. AIAA Paper 93-0669, Jan. 1993.

[81] T. J. KAo, T. Y. SU, AND N. J. YU, Navier-Stokes calculations for transport wing-body configurations with nacelles and struts. AIAA Paper 93-2945, July 1993.

[82] S. R. Kennon, J. M. Meyering, C. W. Berry, And J. T. Oden, Geometry based Delaunay tetrahedralization and mesh movement strategies for multi-body CFD. AIAA Paper 92-4575, Aug. 1992.

[83] W. L. Kleb, J. T. Batina, And M. H. Wildiams, Temporal adaptive Euler/Navier-Stokes algorithm involving unstructured dynamic meshes, AIAA J., 30 (1992), pp. 1980-1985.

[84] D. D. KNIGHT, A fully implicit Navier-Stokes algorithm using an unstructured grid and flux -difference splitting. AIAA Paper 93-0875, Jan. 1993. 
[85] B. Koobus, M. H. Lallemand, And A. Dervieux, Unstructured volume-agglomeration mg: Solution of the poisson equation. INRIA Report 1946, June 1993.

[86] R. E. LaBarre, Computational Geometry Techniques for $2 D$ and $3 D$ Unstructured Mesh Generation with Application to the Solution of Divergence Form Partial Differential Equations, PhD thesis, Univ. of Connecticut, 1992.

[87] M. Lallemand, H. Steve, and A. Dervievx, lnstructured multigridding by volume agglomeration: Current status, Computers and Fluids, 21 (1992), pp. 397-433.

[88] P. LAx, Hyperbolic systems of conservation laws and the mathematical theory of shock waves, vol. 11 of SIAM Regional series on Applied Mathematics, ST'AM, Philadelphia, PA, second ed., 1973.

[89] A. LeRAT AND J. Sides, Numerical simulation of unsteady transonic flows using the Euler equations in integral form, Israel J. Tech., 17 (1979), pp. 302-310.

[90] F. W. LETNIOWSKI, Three-dimensional delaunay triangulations for finite element approximations to a second order diffusion operator, SIAM J. Sci. Stat. Comput., 13 (1992), pp. 765-770.

[91] R. J. Leveque, Numerical methods for conservation laws, Birkhauser Press, Germany, 1992.

[92] M.-S. Liou And C. J. Steffan, A new flux splitting scheme, J. Comp. Phys., 107 (1993), pp. 23-39.

[93] R. LöHNER, An adaptive finite element solver for transient problems with moving bodies, Comp. Struct., 30 (1988), pp. 303-317.

[94] R. LöhNER AND J. D. BAUM, Adaptive H-refinement on 3-D unstructured grids for transient problems, Int. J. Num. Meth. Fluids, 14 (1992), pp. 1407-1419.

[95] R. Löhner, K. Morgan, J. Peraire, and M. VAhDati, Finite element flux-corrected transport (FCT) for the Euler and Navier-Stokes equations, Comput. Methods Appl. Mech. Engrg., 7 (1987), pp. 1093-1109.

[96] R. Löhner, K. Morgan, AND O. Zienkiewicz, An adaptive finite element procedure for compressible high speed flows, Comput. Methods Appl. Mech. Engrg, 51 (1985), pp. 441-465.

[97] H. LUO, J. D. BAUM, AND R. LÖHNER, Edge-based finite element scheme for the Euler equations, AIAA J., 32 (1994), pp. 1183-1190.

[98] H. Luo, J. D. Baum, R. Löhner, And J. Cabello, Adaptive edge-based finite element schemes for the Euler and Navier-Stokes equations on unstructured grids. AIAA Paper 93-0336, Jan. 1993.

[99] - Implicit schemes and boundary conditions for compressible flows on unstructured meshes. AIAA Paper 94-0816, Jan. 1994.

[100] D. L. Marcum AND R. K. Agarwal, A three-dimensional finite element Navier-Stokes solver with $k-\epsilon$ turbulence model for unstructured grids. AIAA Paper 90-1652, June 1990.

[101] D. J. Mavriplis, A three-dimensional multigrid Reynolds-averaged Navier-Stokes solver for unstructured meshes. AIAA Paper 94-1878, June 1994; to appear in AIAA J.

[102] — Accurate multigrid solution of the Euler equations on unstructured and adaptive meshes, AIAA J., 28 (1990), pp. 213-221. 
[103] —- Adaptive mesh generation for viscous flows using Delaunay triangulation, J. Comp. Phys., 90 (1990), pp. 271-291.

[104] —, Algebraic turbulence modeling for unstructured and adaptive meshes, AIAA J., 29 (1991), pp. 2086-2093.

[105] —, Three-dimensional multigrid for the Euler equations, AIAA Journal, 30 (1992), pp. 17531761.

[106] - Mesh generation and adaptivity for complex geometries, Handbook of Computational Fluid Mechanics, Academic Press, Inc., San Diego, CA, 1995.

[107] D. J. Mavriplis, R. DAS, J. Saltz, and R. E. Vermelaxd, Implementation of a parallel unstructured Euler solver on shared and distributed memory machines. ICASE Report No. 92-68, 1992; to appear in The J. of Supercomputing.

[108] D. J. Mavriplis and V. Venkatakrishnan, Agglomeration multigrid for viscous turbulent flows. AIAA Paper 94-2332, June 1994; to appear in Computers and Fluids.

[109] - A $3 D$ agglomeration multigrid solver for the Reynolds-averaged Navier-Stokes equations on unstructured meshes. AIAA Paper 95-0345, Jan. 1995.

[110] R. L. MEAKIN, Moving body overset grid methods for complete aircraft tiltrotor simulations. AIAA Paper 93-3350CP, July 1993.

[111] J. A. Meijerink AND H. A. van DER Vorst, Guidelines for the usage of incomplete decompositions in solving sets of linear equations as they occur in practical problems, J. Comp. Phys., 44 (1981), pp. 134-155.

[112] N. D. Melson, M. D. Sanetrik, and H. L. Atkins, Time-accurate Navier-Stokes calculations with multigrid acceleration, in 6th Copper Mountain Conf. on Multigrid Methods, 1993, pp. 423439.

[113] J. E. Melton, S. A. Pandya, And J. L. Steger, 3D Euler flow solutions using unstructured Cartesian and prismatic grids. AIAA Paper 93-0331, Jan. 1993.

[114] G. L. Miller, S.-H. Teng, W. Thurston, and S. A. Vavasis, Automatic mesh partitioning, in Graph Theory and Sparse Matrix Computation, A. George, J. R. Gilbert, and J. W. H. Liu, eds., Springer Verlag, 1993, pp. 5i-84. The IMA Volumes in Mathematics and its Applications, Volume 56 .

[115] C. R. MITCHELL, Improved reconstruction schemes for the Navier-Stokes equations on unstructured meshes. AIAA Paper 94-0642, Jan. 1994.

[116] B. Mohammad, Complex turbulent compressible flow computation using a two-layer approach, Int. J. Num. Meth. Fluids, 15 (1992), pp. $747-771$.

[117] K. NAKAHASHI, A finite-element method on prismatic elements for the three-dimensional NavierStokes equations, in Lecture Notes in Physics, vol. 323, Springer Verlag, 1989.

[118] K. NAKAHASHI AND K. EGAMI, An automatic Euler solver using the unstructured upuind method, Computer and Fluids, 19 (1991), pp. 273-286.

[119] R. H. NI, A multiple-grid scheme for solving the Euler equations, AIAA J., 20 (1982), pp. 15651571. 
[120] S. OSHER, Riemann solvers, the entropy condition and difference approximations, SIAM J. Numer. Anal., 21 (1984), pp. 21i-235.

[121] H. Paillere, H. Deconinck, R. Struijs, P. L. Roe, L. M. Mesaros, and J. D. Muller, Computations of compressible flows using fluctuation-splitting on triangular meshes. AIAA Paper 93-3301CP, July 1993.

[122] B. PAlmerio, An attraction-repulsion mesh adaption model for flow solution on unstructured grids, Computers and Fluids, 23 (1994). pp. 487-506.

[123] M. Paraschivoiu, J. Y. Trepanier, M. Reggio, and R. Camarero, A conservative dynamic discontinuity tracking algorithm for the Euler equations. AIAA Paper 94-0081, Jan. 1994.

[124] P. Parikh, R. Löhner, C. Gumbert, and S. Pirzadeh, Numerical solutions on a PATHFINDER and other configura tions using unstructured grids and a finite element solver. AIAA Paper 89-0362, Jan. 1989.

[125] I. PARPIA AND D. J. MICHALAK, Grid-independent upwind scheme for multidimensional flows, AIAA J., 31 (1993), pp. 646-651.

[126] I. PARPIA AND P. PARIKH, A solution-adaptive mesh generation method with cell-face orientation control. AIAA Paper 94-0416, Jan. 1994.

[127] V. Parthasarathy AND Y. Kallinderis, New multigrid approach for three-dimensional unstructured, adaptive grids, AIAA J., 32 (1994), pp. 956-963.

[128] V. Parthasarathy, Y. Kallinderis, and K. Nakajima, Hybrid adaptation method and directional viscous multigrid with prismatic-tetrahedral meshes. AIAA Paper 95-0670, 1995.

[129] J. Peraire, J. Peirö, and K. Morgan, A $3 d$ finite-element multigrid solver for the Euler equations. AIA A Paper 92-0449, Jan. 1992.

[130] _ - Adaptive remeshing for three-dimensional compressible flow computations, J. Comp. Phys., 103 (1992), pp. 269-285.

[131] E. PEREZ, A 3 d finite-element multigrid solver for the Euler equations. INRIA Report No. 442, Sept. 1985.

[132] S. PIRZADEH, Viscous unstructured three-dimensional grids by the Advancing-Layers Method. AIAA Paper 94-0417, Jan. 1994.

[133] A. Pothen, H. D. Simon, AND K. P. Liou, Partitioning sparse matrices with eigenvectors of graphs, SIAM J. Matrix Anal. Appl., 11 (1990), pp. 430-452.

[134] M. Potsdam, G. Intemann, N. T. Frink, R. Campbell, L. Smith, and S. Pirzadeh, Wing pylon fillet design using unstructured mesh Euler solvers. AIA A Paper 93-3500, Aug. 1993.

[135] K. G. Powell, P. L. RoE, AND J. J. Quirk, Adaptive-mesh algorithms for computational fluid dynamics, in Algorithmic Trends in Computational Fluid Dynamics, M. Y. Hussaini, A. Kumar, and M. D. Salas, eds., New York, 1992, Springer Verlag, pp. 301-337.

[136] T. H. Pulliam, Time accuracy and the use of implicit schemes. AIAA Paper 93-3360CP, July 1993.

[137] J. J. QUIRK, A cartesian approach with hierarchical refinement for compressible flows, in Computational Fluid Dynamics, S. Wagner et al., ed., New York, 1994, John Wiley and Sons, pp. 200-209. 
[138] — Parallel adaptive grid algorithm for computational shock hydrodynamics. Submitted for publication to Appl. Num. Math., 1994.

[139] M. M. RaI, Navier-Stokes simulations of blade-vortex interaction using high-order accurate upwind schemes. AIAA Paper 87-0543, Jan. 1987.

[140] R. Ramamurthi, W. Sandberg, and R. Löhner, Evaluation of a scalable 3-D finite element incompressible flow solver. AIAA Paper 94-0756, Jan. 1994.

[141] R. D. Rausch, J. T. Batina, And H. T. Y. YAng, Spatial adaptation of unstructured meshes for unsteady aerodynamic flow computations, AIAA J., 30 (1992), pp. 1243-1251.

[142] P. L. Roe, Approximate Riemann solvers, parameter vectors and difference schemes, J. Comp. Phys., 43 (1981), pp. 35i-3i2.

[143] — Characteristic-based schemes for the Euler equations, Ann. Rev. Fluid Mech., 18 (1986), pp. $337-365$.

[144] - Discrete models for the numerical analysis of time-dependent multidimensional gas dynamics, J. Comp. Phys., 63 (1986). pp. 458-476.

[145] - Finite-volume methods for the compressible Navier-Stokes equations, in Numerical methods for laminar and turbulent flows - Part 2, C. Taylor, W. G. Habashi, and M. M. Hafez, eds., Montreal, Canada, 1987, Pineridge Press.

[146] —, Beyond the Riemann problem, Part I, in Algorithmic Trends in Computational Fluid Dynamics, M. Y. Hussaini, A. Kumar, and M. D. Salas, eds., New York, 1992, Springer Verlag, pp. $341-367$.

[147] P. Rostand and B. Stoufflet, TVD schemes to compute compressible viscous flows on unstructured meshes, in Notes on numerical fluid mechanics, vol. 24, Braunschweig, Germany, 1988, Vieweg Press.

[148] C. L. Rumsey, B. van LeEr, and P. L. Roe, A multidimensional flux function with applications to the Euler and Navier-Stokes equations, J. Comp. Phys., 105 (1993), pp. 306-323.

[149] Y. SaAd AND M. H. Schuttz, Gmres: A generalized minimal residual algorithm for solving nonsymmetric linear systems, SIAM J. Sci. Stat. Comput., 7 (1986), pp. 856-869.

[150] V. SELMIN, The node-centered finite volume approach: Bridge between finite differences and finite elements, Comput. Methods Appl. Mech. Engrg, 102 (1993), pp. 107-138.

[151] F. SHAKIB, T. J. R. Hughes, AND Z. JohAN, A multi-element group preconditioned gmres algorithm for nonsymmetric problems arising in finite element analysis, Comput. Methods Appl. Mech. Engrg, 87 (1989), pp. 415-456.

[152] R. A. Shapiro ANd E. M. Murman, Higher-order and 3-d finite element methods for the Euler equations. AIAA Paper 89-0655, Jan. 1989.

[153] C. SHU, TVB uniformly high-order schemes for conservation laws, Math. Comp., 49 (1987), pp. 105-121.

[154] D. Sidilkover, A genuinely multidimensional upwind scheme and efficient multigrid solver for the compressible Euler equations, (1994). Submitted for publication to J. Comp. Phys.

[155] D. Sidilkover AND P. L. Roe, Unification of some advection schemes in two dimensions. In preparation for ICASE Report, 1995. 
[156] H. D. Simon, Partitioning of unstructured problems for parallel processing, Computing Systems in Engrg., 2 (1991), pp. 135-148.

[157] D. C. Slack, D. L. Whitaker, and R. W. Walters, Time integration algorithms for the two-dimensional Euler equations on unstructured meshes, AIAA J., 32 (1994), pp. 1158-1166.

[158] W. A. SMiтh, Multigrid solution of transonic flow on unstructured grids, in Recent Advances and Applications in Computational Fluid Dynamics, Nov. 1990. Proceedings of the ASME Winter Annual Meeting, Ed. O. Baysal.

[159] P. R. Spalart AND S. R. Allmaras, A one-equation turbulence model for aerodynamic flows. AIAA Paper 92-0439, Jan. 1992.

[160] S. P. SPEKREIJSE, Multigrid discretization of monotone second-order discretizations of hyperbolic conservation laws, Math. Comp., 45 (1987), pp. 135-155.

[161] J. L. STEGER, Implicit finite-difference simulation of flow about arbitrary two-dimensional geometries, AIAA J., 17 (1978), pp. 679-686.

[162] S. Tatsumi, L. Martinelli, and A. Jameson, Design, implementation, and validation of flux limited schemes for the solution of the compressible Navier-Stokes equations. AIAA Paper 94-064i, Jan. 1994.

[163] R. R. Thareja, J. R. Stewart, O. Hassan, K. Morgan, and J. Peraire, A point implicit unstructured grid solver for the Euler and Navier-Stokes equations. AIAA Paper 88-0036, Jan. 1988.

[164] J. L. Thomas, S. L. TAYLOR, AND W. K. ANDERSOn, Navier-Stokes computations of vortical flows over low aspect ratio wings. AIAA Paper 87-0207, Jan. 1987.

[165] P. D. Thomas AND C. K. Lombard, Geometric conservation law and its applications to flow computations on moving grids, AIAA J., 17 (1979), pp. 1030-1037.

[166] J. F. THOMPSON AND N. P. WEATHERILL, Aspects of numerical grid generation: current science and art. AIAA-93-3539-CP, 1993.

[167] J. Y. Trepanier, H. Zhang, M. Reggio, and R. Camarero, Adaptive and moving meshes for the computation of unsteady compressible flows, in Numerical grid generation in Computational Fluid dynamics and related fields, A. S. Arcilla, J. Hauser, P. R. Eiseman, and J. F. Thompson, eds., New York, 1991, North-Holland, pp. 43-54.

[168] W. O. VAlERzo AND D. J. MAVRIPLIS, Navier-Stokes applications to high-lift airfoil analysis. AIAA Paper 93-3534, Aug. 1993.

[169] B. VAN LEER, Towards the ultimate conservative difference scheme $V$. A second order sequel to Godunov's method, J. Comp. Phys., 32 (1979), pp. 101-136.

[170] B. van LeER, C. H. TAI, AND K. G. Powell, Design of optimally-smoothing multi-stage schemes for the Euler equations. AIAA Paper 89-1933, June 1989.

[171] J. VAN Rosendale, Floating point shock fitting for the Euler equations. Paper submitted to the 12th AIA A CFD Conf., San Diego, CA., June 1995.

[172] J. C. Vassberg, A fast, implicit unstructured-mesh Euler method. AIAA Paper 92-2693, 1992.

[1i3] V. N. Vatsa, M. D. Sanetrik, and E. B. Parlette, Development of a flexible and efficient multigrid-based multi-block flow solver. AIAA Paper 93-0677, 1993. 
[174] V. VENKATAKRISHNAN, On the accuracy of limiters and convergence to steady state solutions. AIAA Paper 93-0880, Jan. 1993; to appear in J. Comp. Physics.

[175] - Parallel implicit unstructured grid Euler solvers, AIAA J., 32 (1994), pp. 1985-1991.

[176] V. Venkatakrishnan and T. J. Barth, Application of direct solvers to unstructured meshes for the Euler and Navier-Stokes equations using upwind schemes. AIAA Paper 89-0364, Jan. 1989.

[17i] V. VEnKatakrishNAN AND D. J. MAVRIPLIS, Agglomeration multigrid for the threc-dimensional Euler equations. AIAA Paper 94-0069, June 1994; to appear in AIAA J.

[178] - Implicit solvers for unstructured meshes, Journal of Computational Physics, 105 (1993), pp. 83-91.

[179] - Implicit method for the computation of unsteady flows on unstructured grids. Paper submitted to the 12th AIAA CFD Conf., San Diego, CA., June 1995.

[180] V. Venkatakrishnan, H. D. Simon, and T. J. Barth, A mimd implementation of a parallel Euler solver for unstructured grids, The J. of Supercomputing, 6 (1992), pp. 117-137.

[181] R. VILSMEIER AND D. HÄNEL, Adaptive methods on unstructured grids for Euler and NavierStokes equations, Computers and Fluids, 22 (1993), pp. 485-499.

[182] G. P. Warren, W. K. Anderson, J. L. Thomas, and S. L. Krist, Grid convergence for adaptive methods. AIAA Paper 91-1592CP, July 1991.

[183] D. F. Watson, Computing the n-dimensional Delaunay tessellation with application to Voronoi polytopes, The Computer J., 24 (1981), pp. 167-172.

[184] D. L. Whitaker, Three-dimensional unstructured grid Euler computations using a fully-implicit, upwind method. AIAA Paper 93-3337CP, July 1993.

[185] D. L. WhITAKER, B. Grossman, AND R. LÖHneR, Two-dimensional Euler computations on a triangular mesh using an upwind finite-volume scheme. AIAA Paper 89-0470, Jan. 1989.

[186] H. Zhang, M. Reggio, J. Y. Trepanier, and R. Camarero, Discrete form of the GCL for moving meshes and its implementation in CFD schemes, Computers and Fluids, 22 (1993), pp. 9-23. 


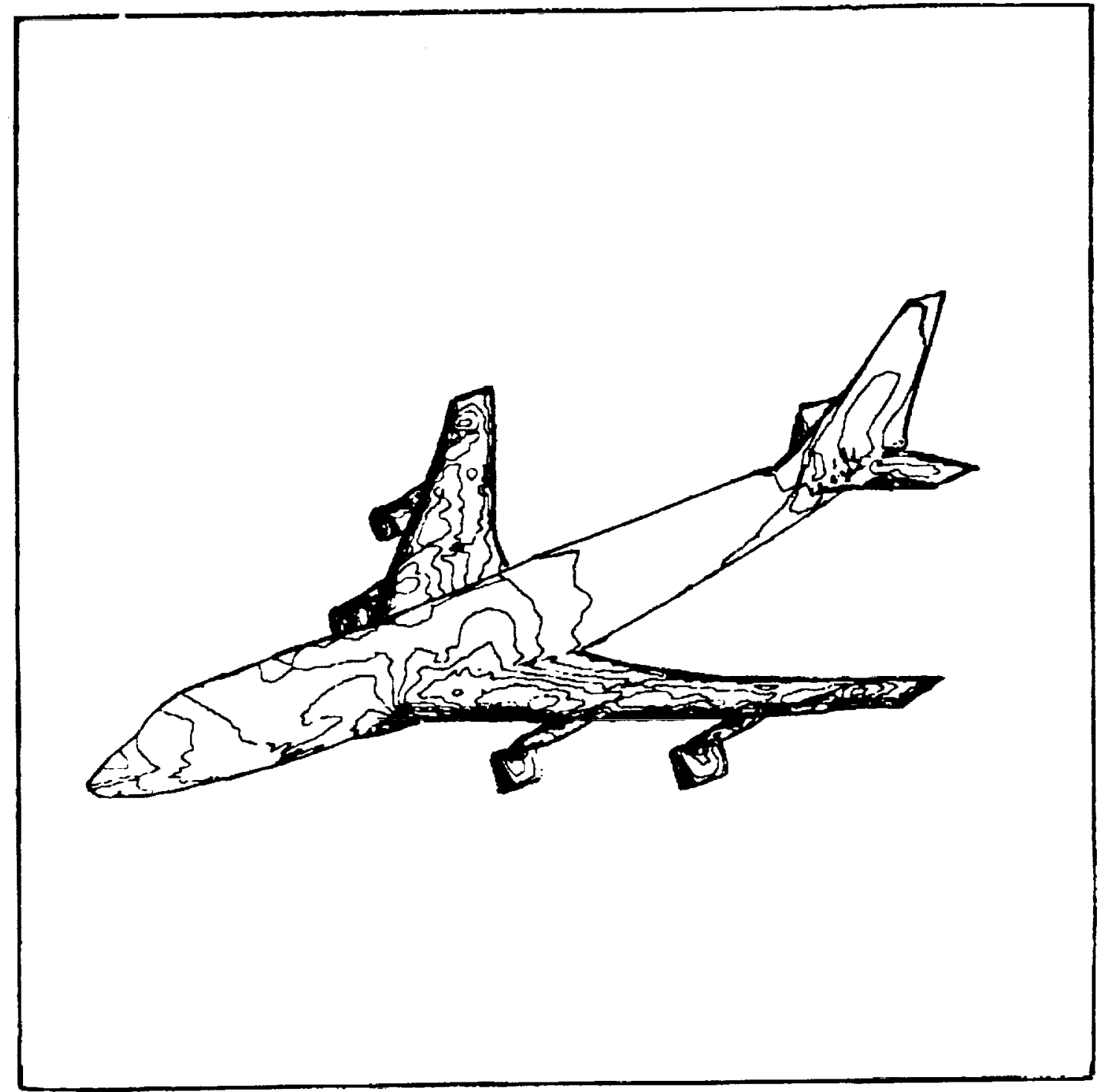

Figure 1. Mach contours for inviscid transonic flow over Boeing 747-200 using an unstructured tetrahedral grid $\left(M_{\infty}=0.8, \alpha=2.73^{\circ}\right)[72]$. 


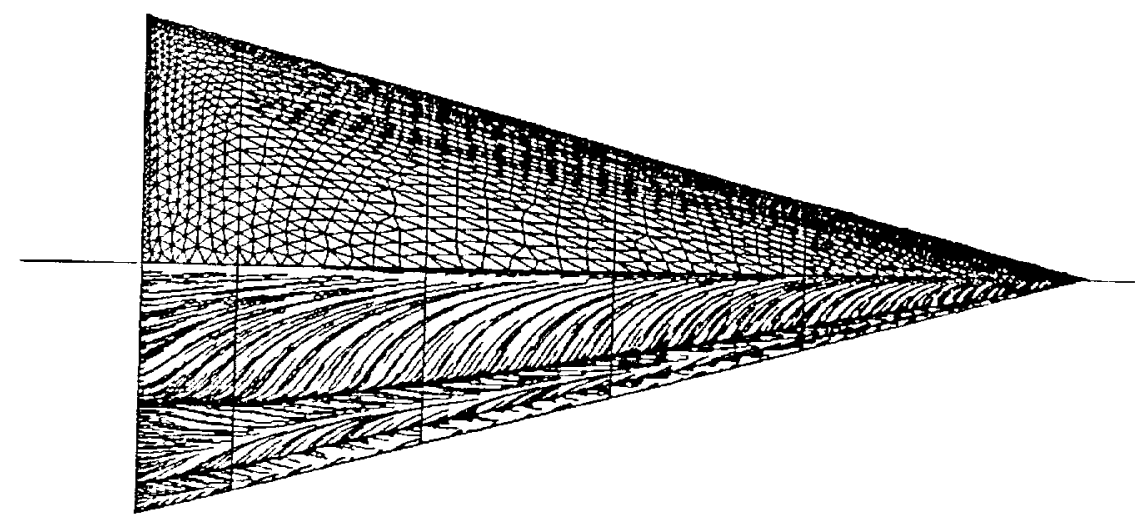

(a)
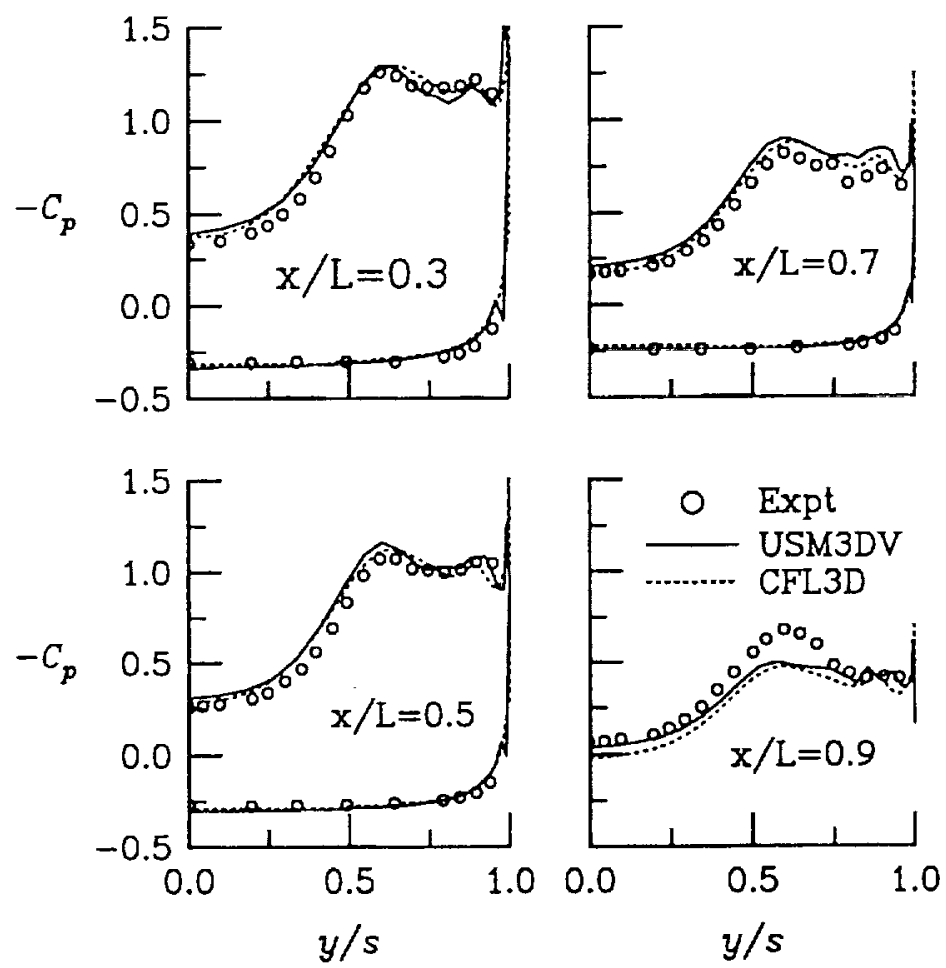

(b)

Figure 2. Laminar flow over a delta wing $\left(M_{\infty}=0.3, \alpha=20.5^{\circ}, R \epsilon=0.9 \times 10^{6}\right)$ [18]. (a) Surface grid and "oil-flow" pattern (b) Pressure profiles at four chord stations. 


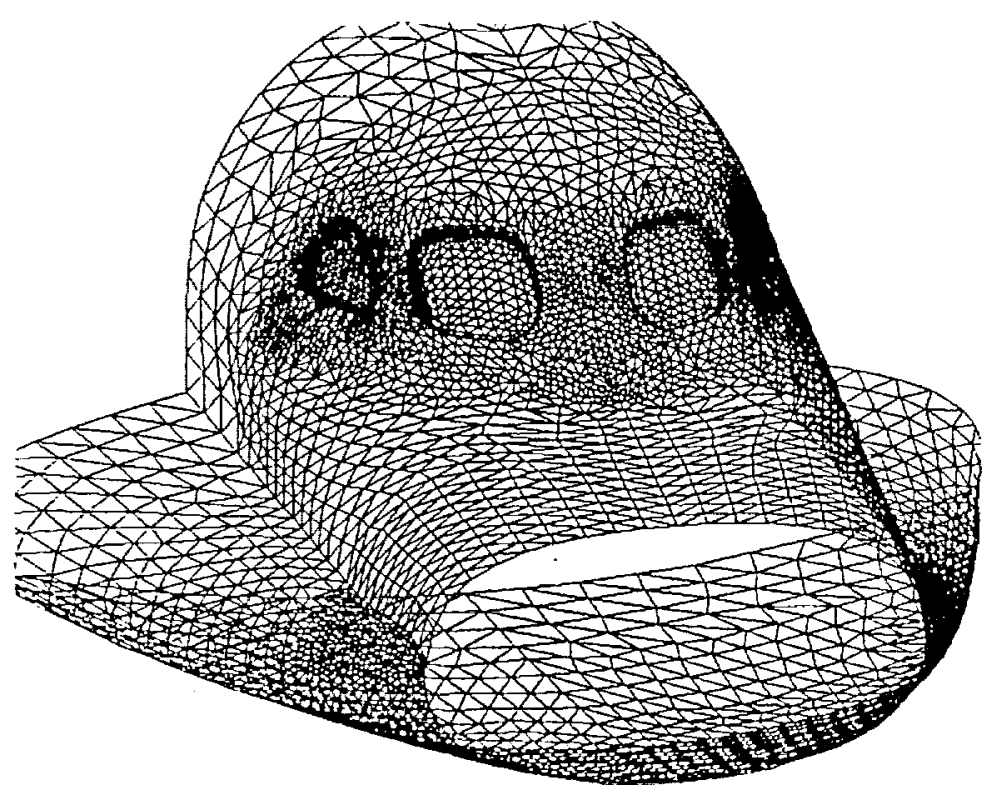

(a)

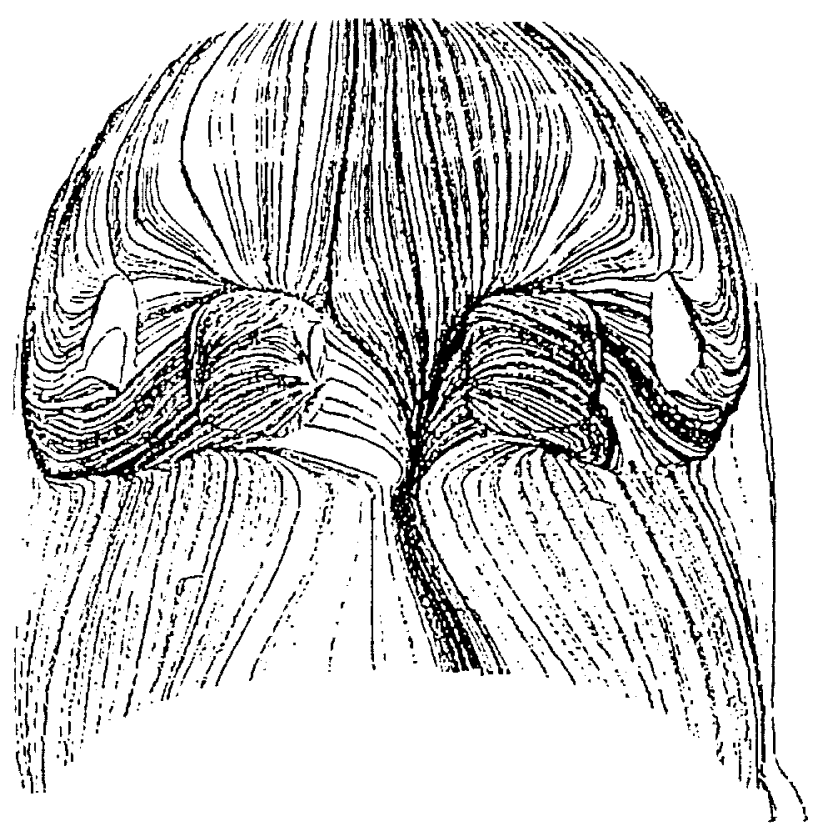

(b)

Figure 3. Hypersonic flow over the canopy of a Hermes [32]. (a) Surface grid. (b). Skin friction lines on the surface. 
Slat

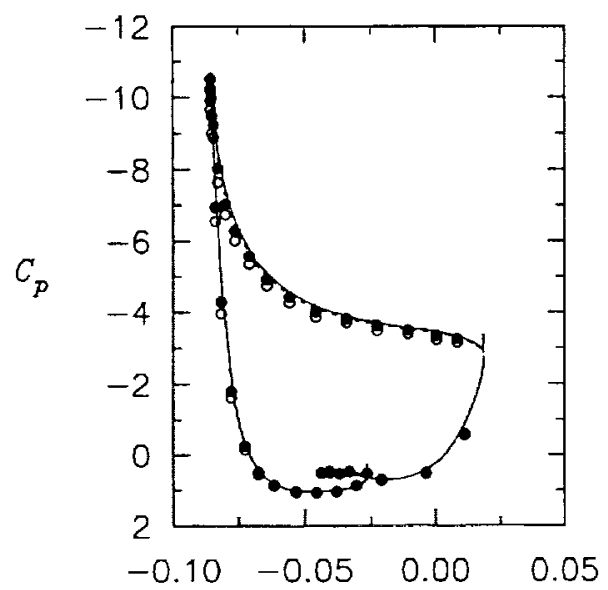

Main

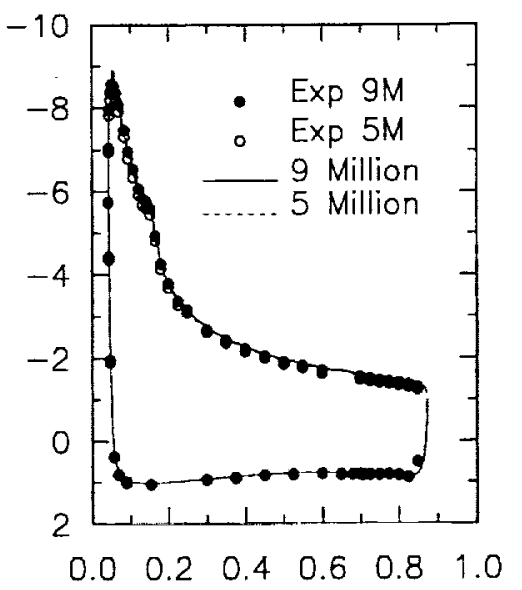

Flap

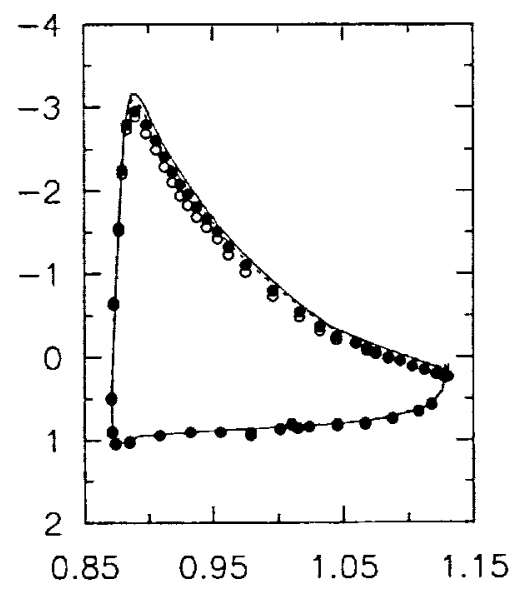

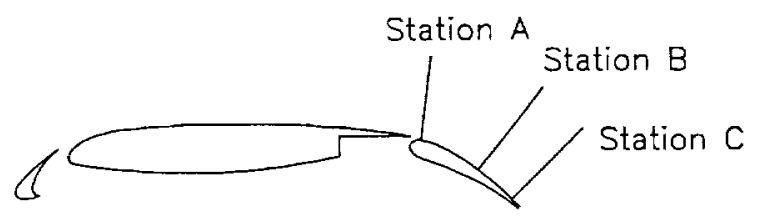

Station A

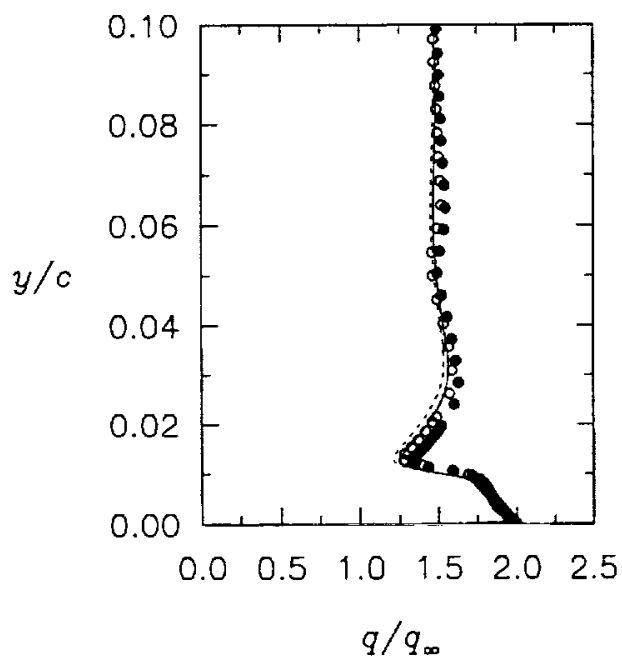

Station B

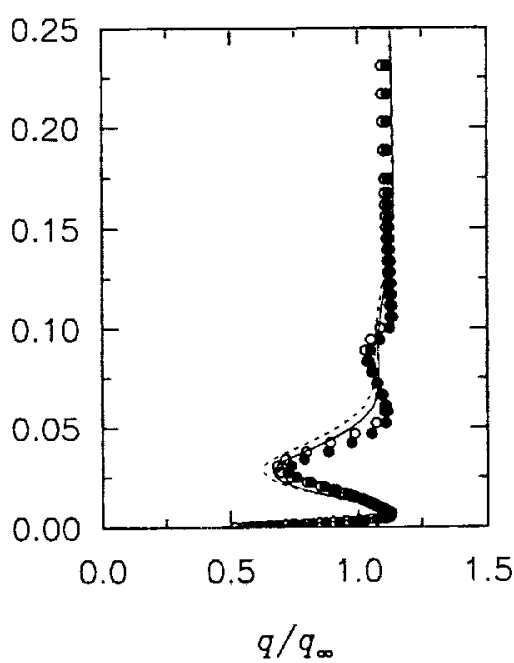

Station C

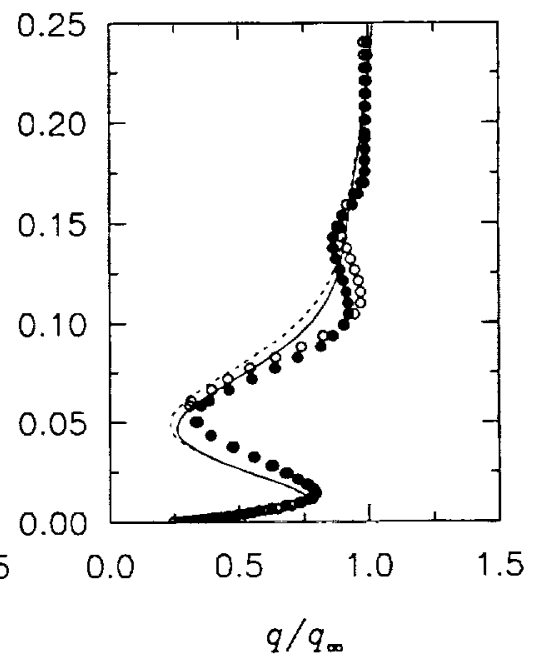

Figure 4. Compressible turbulent flow over a high-lift configuration $\left(M_{\infty}=0.2, \alpha=16^{\circ}\right)[6]$. Surface pressure distributions and velocity profiles at various stations. 


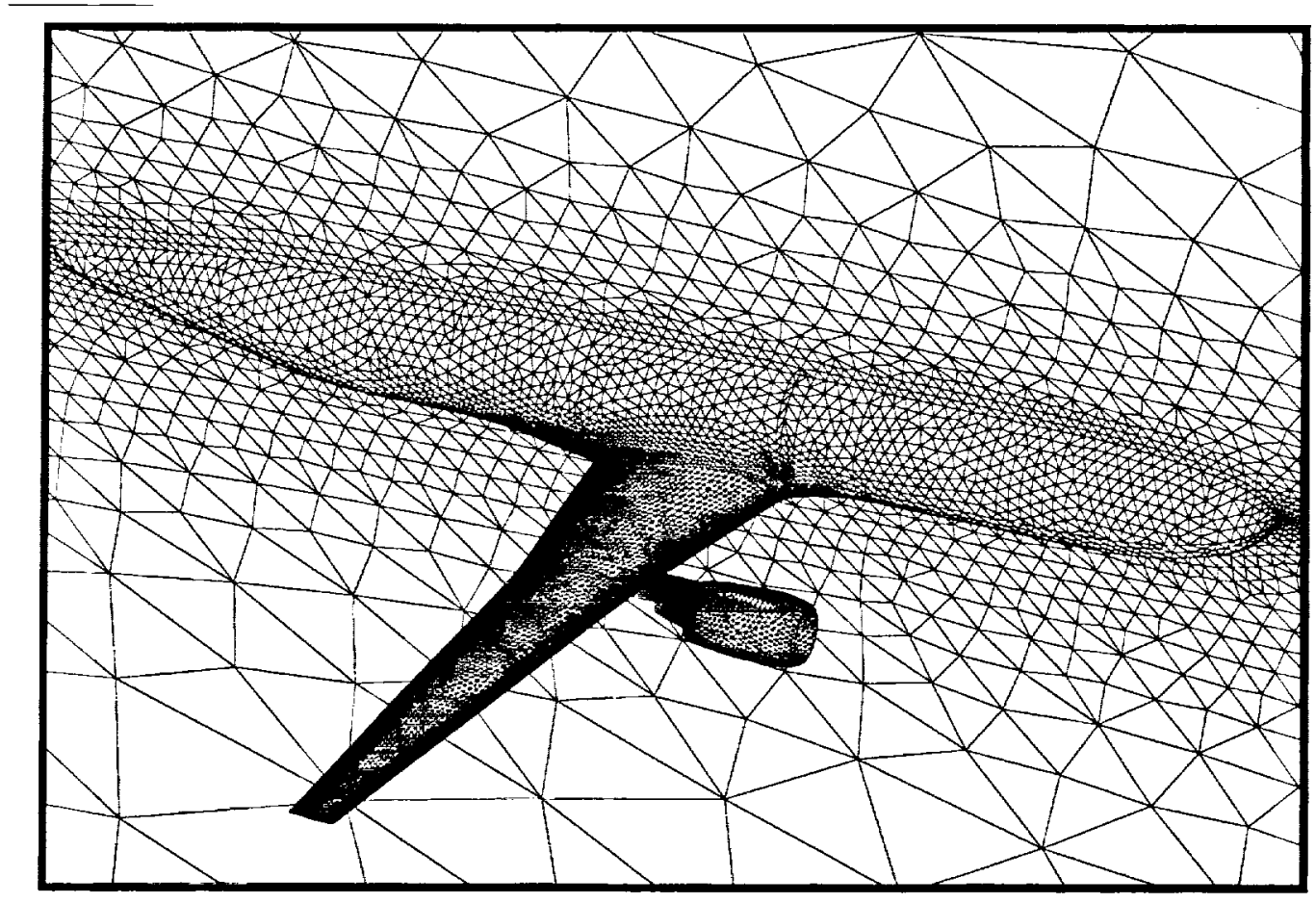

Fig. 5a.

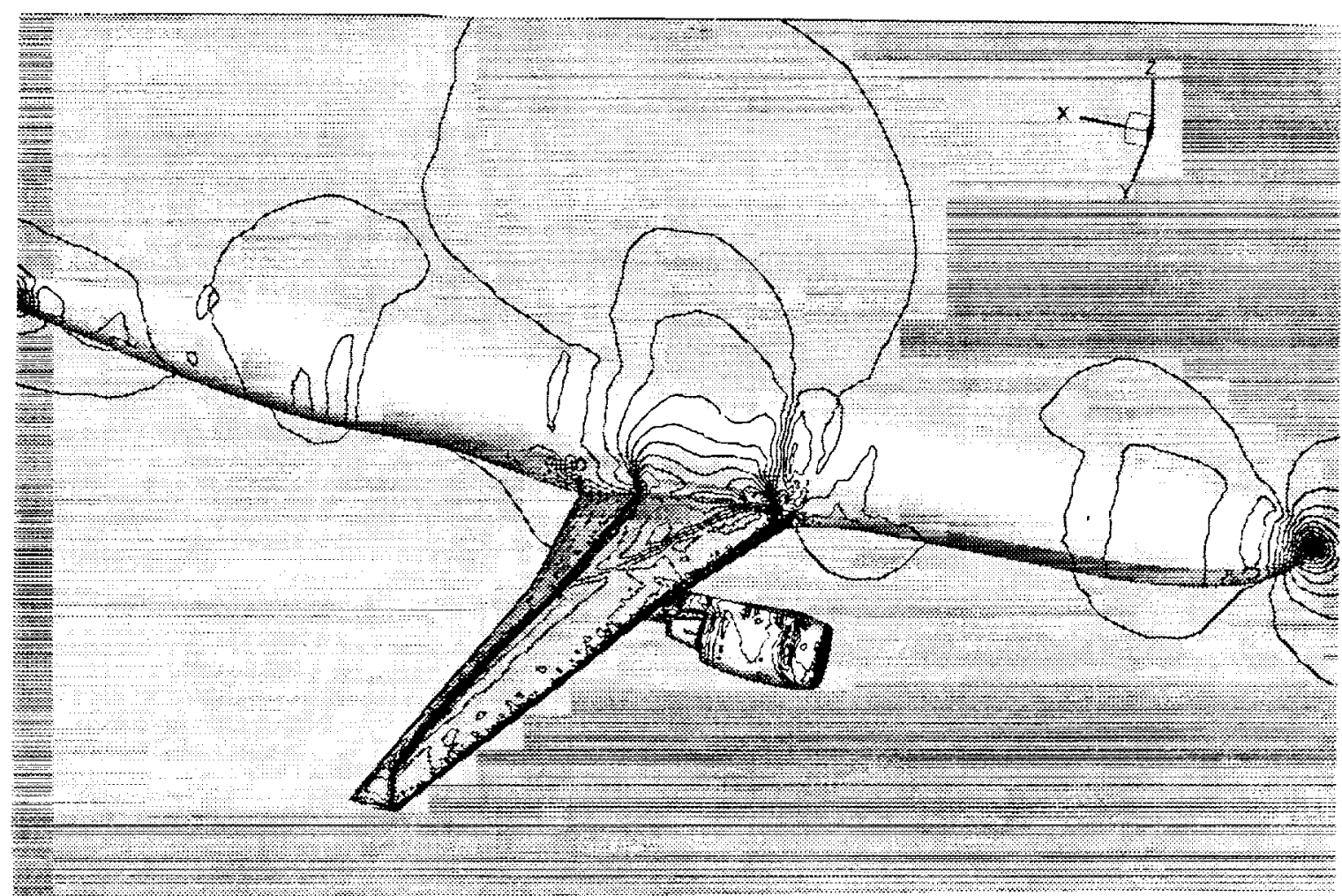

Fig. 5b. 


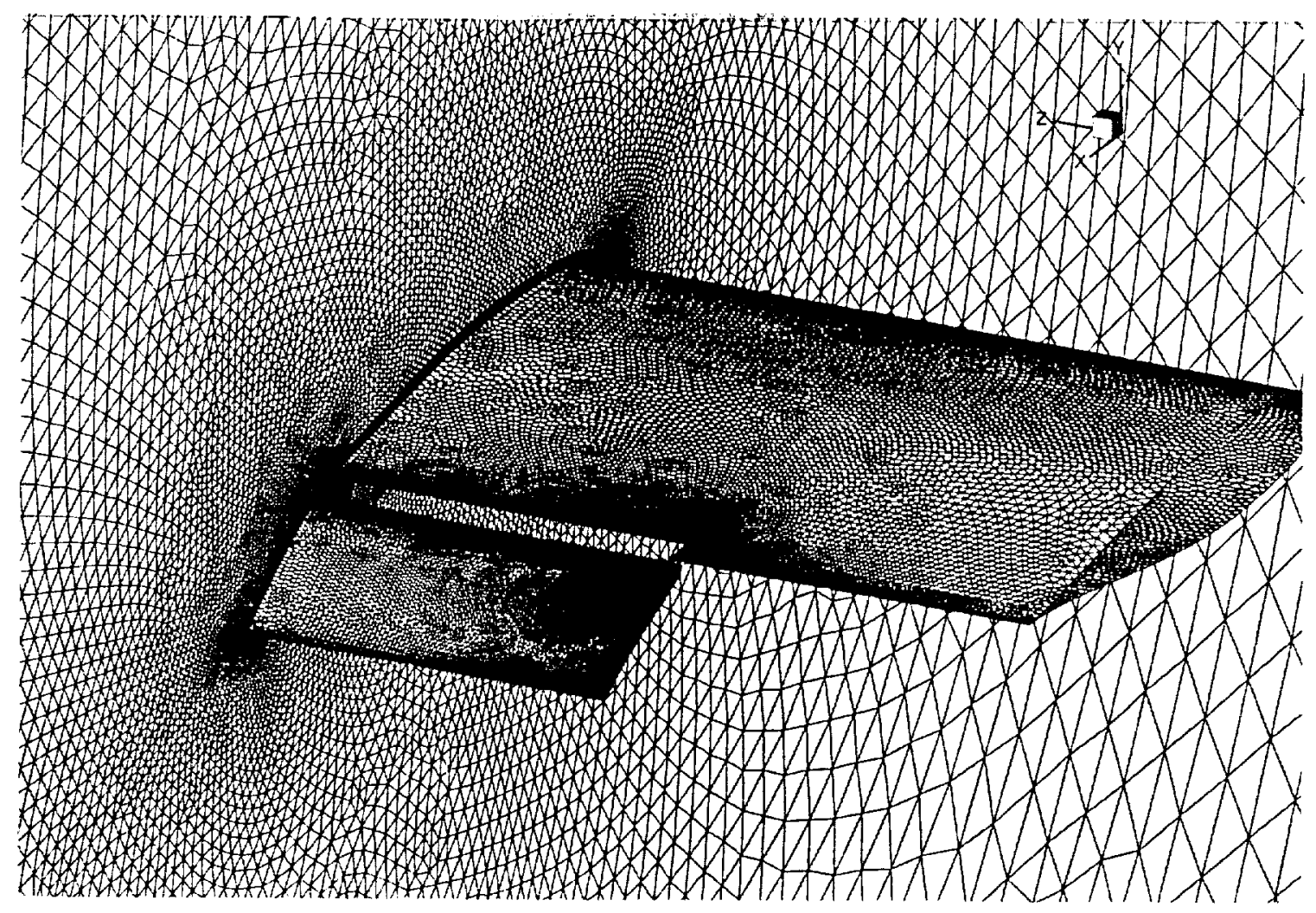

Fig. 5c.

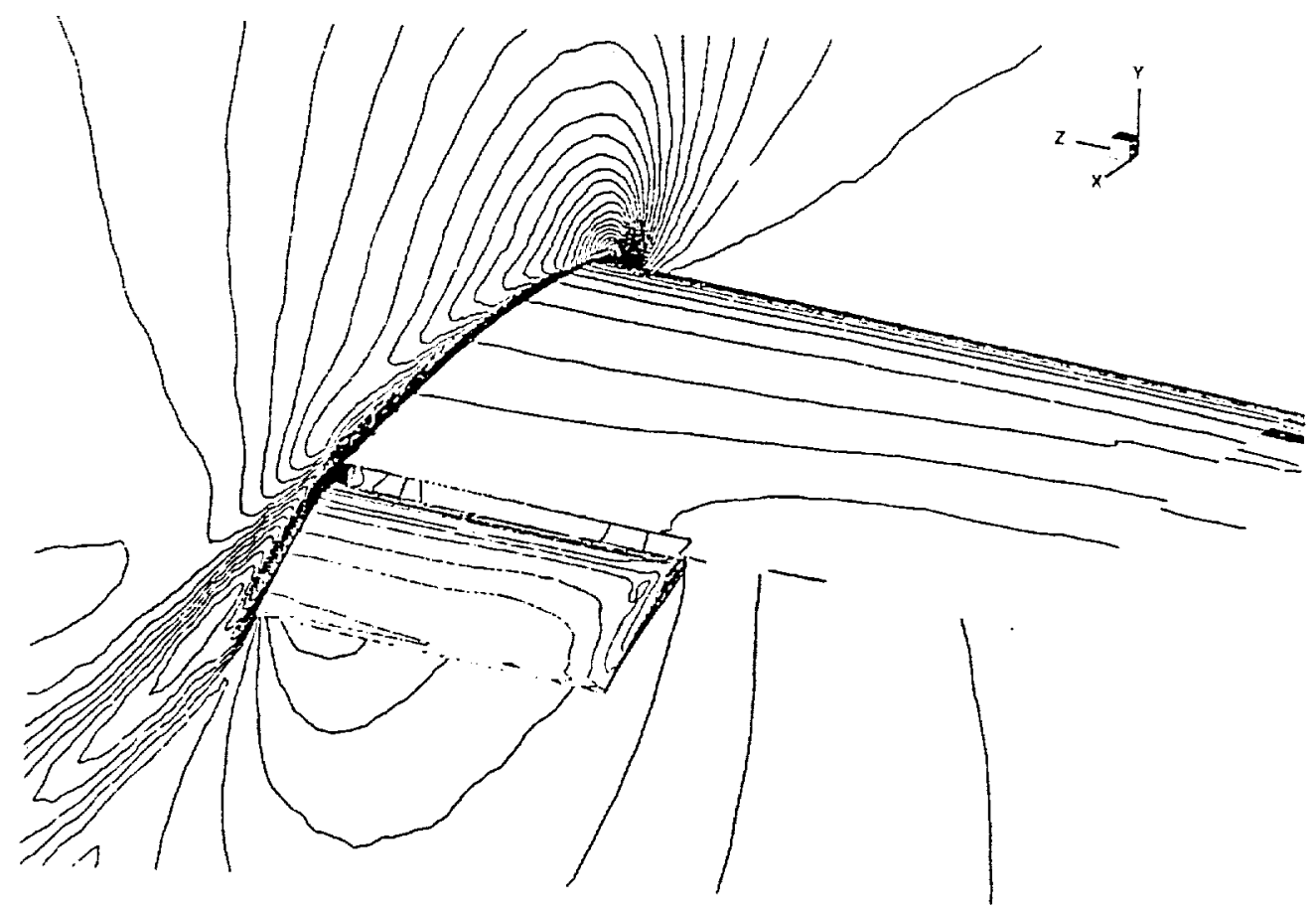

Fig. 5d. 


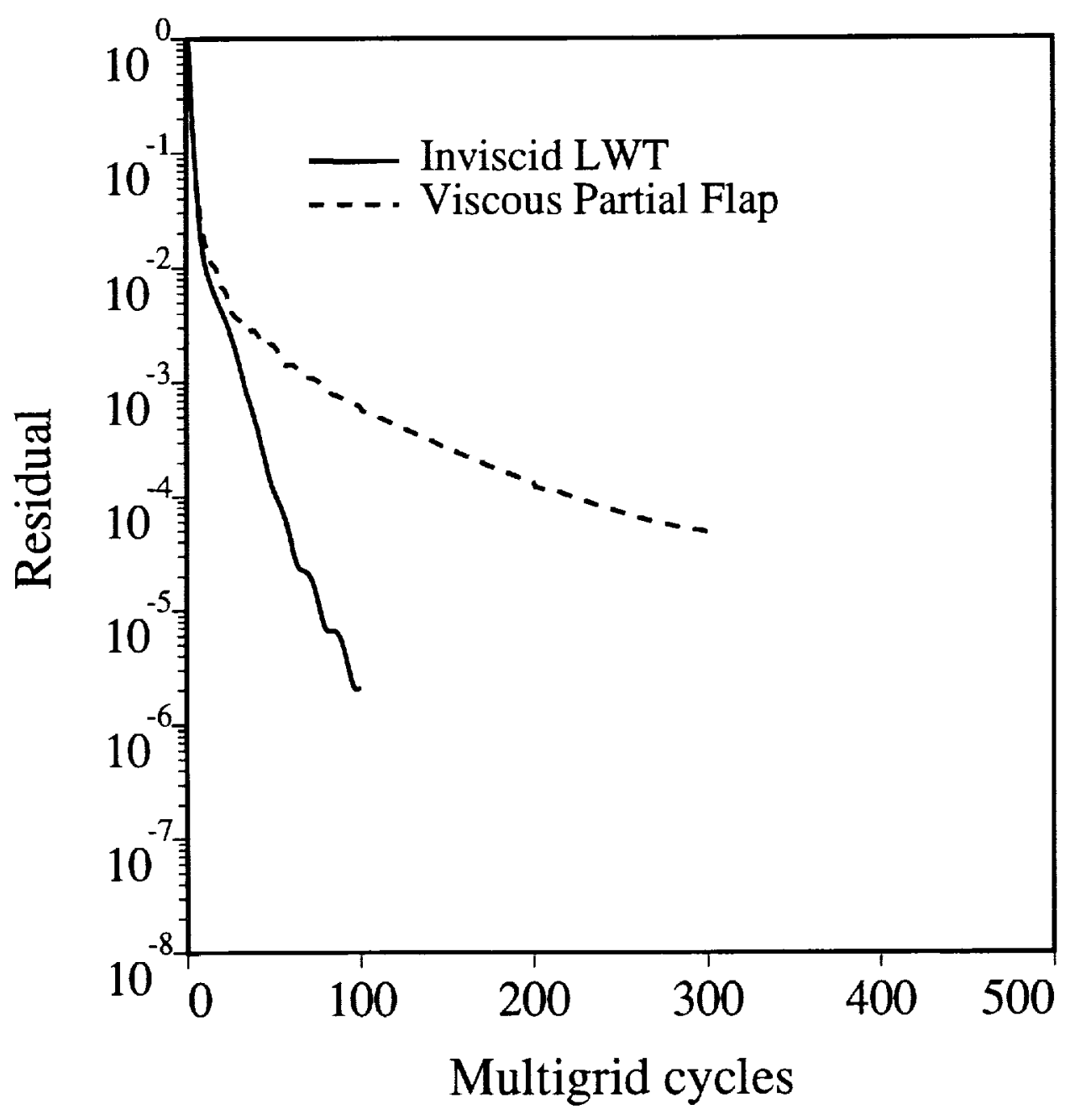

Fig. 5e.

Figure 5. Computations done using the agglomeration multigrid strategy [17 7,109$]$. (a) Surface grid for the low wing transport (LWT) case. (b) Mach contours on the surface for inviscid transonic flow over the LWT. (c) Surface grid for the partial span-flap experimental configuration. (d) Mach contours on the wind-tunnel wall and density contours on the surface for turbulent flow. (e) Convergence histories for the inviscid and the viscous cases. 


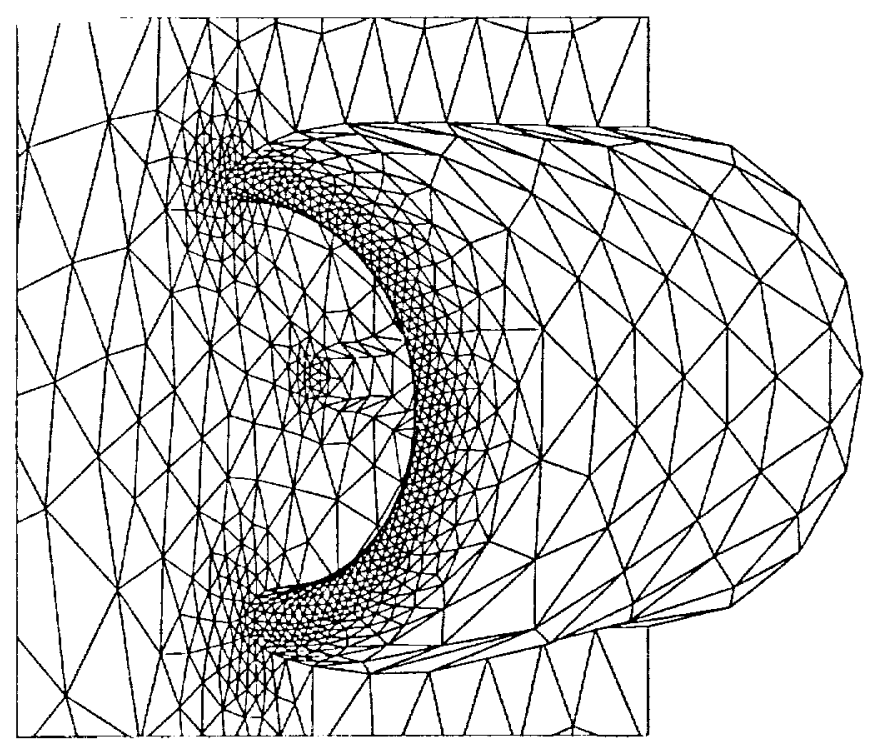

(a)

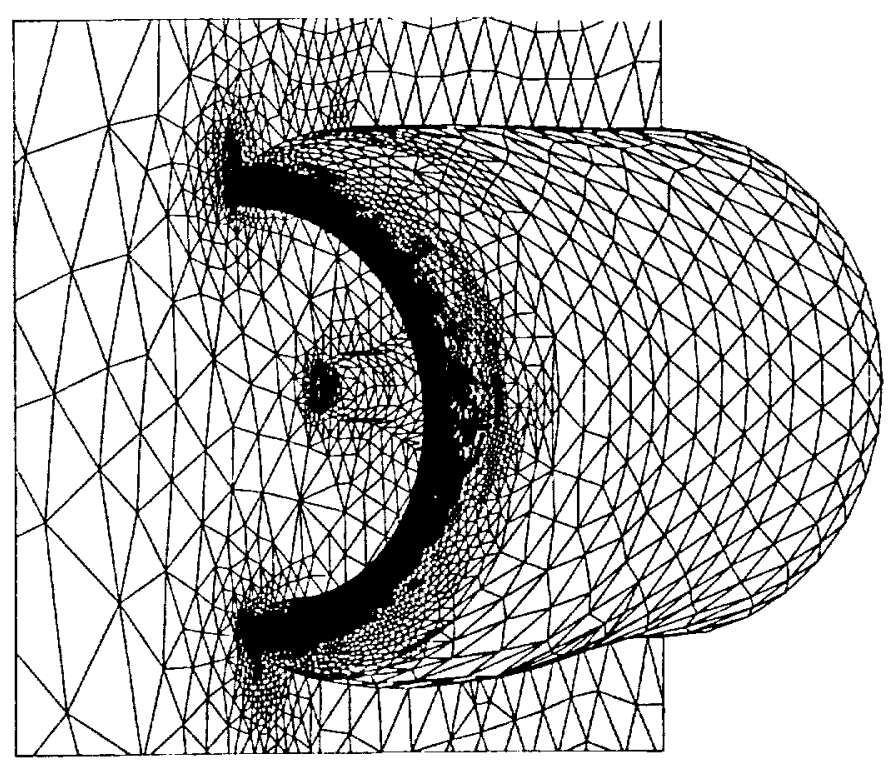

(b)

Figure 6. (a) Initial surface grid for an engine inlet. (b) Girid obtained through adaptive refincment [35]. 


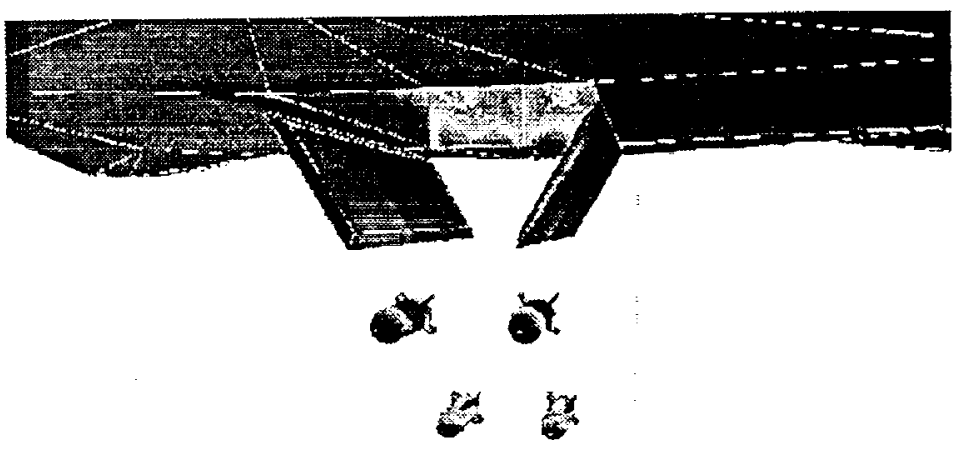

(a)

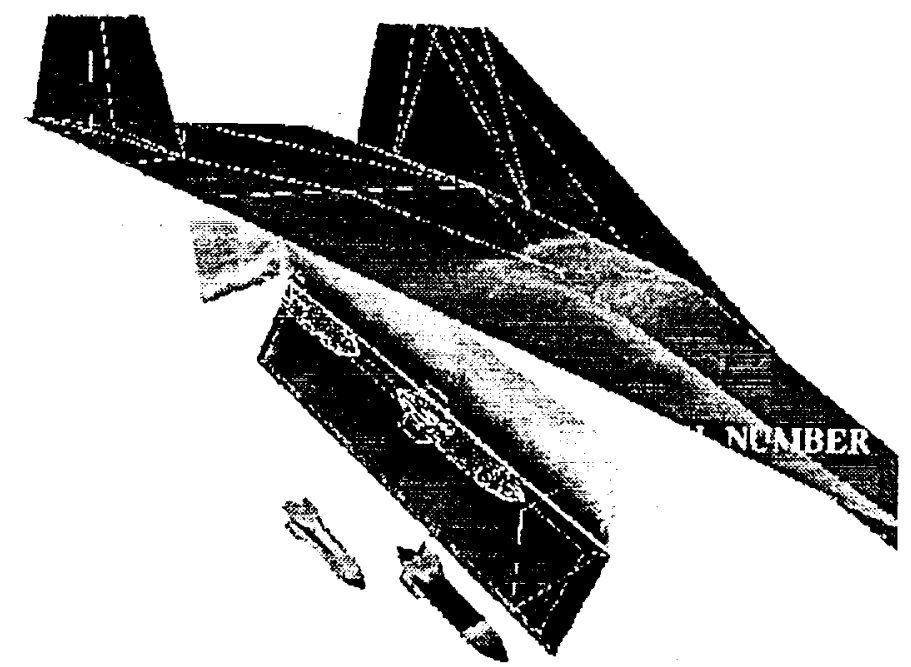

(b)

Figure $i$. Store separation from F-11 7 [18]. Solution at a particular instant in time. (a) Mach contours from outside. (b) Mach contours from inside the bay. (c) Pressure contours illustrating the shock wave patterns about the projectiles. 


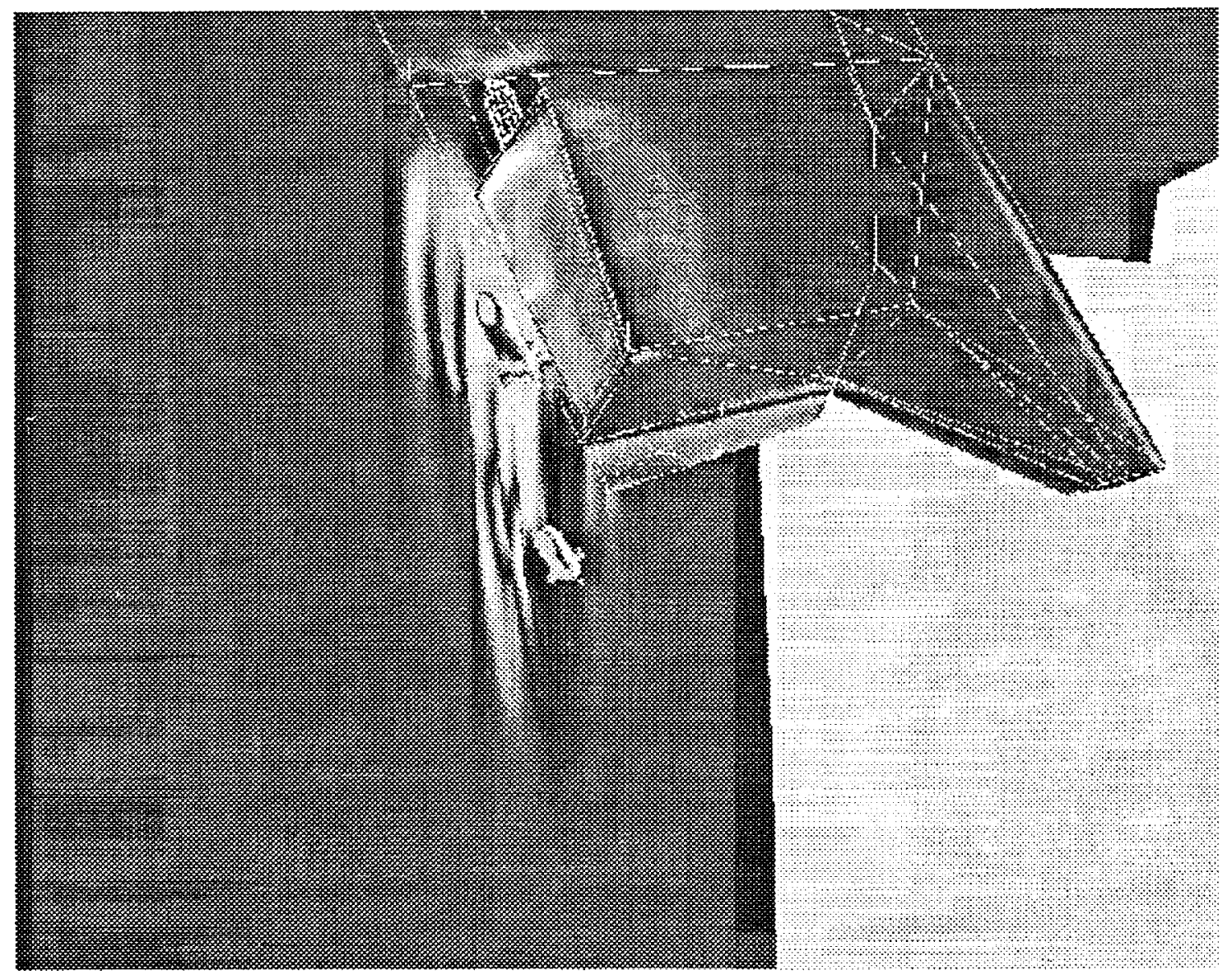

Fig. ic. 


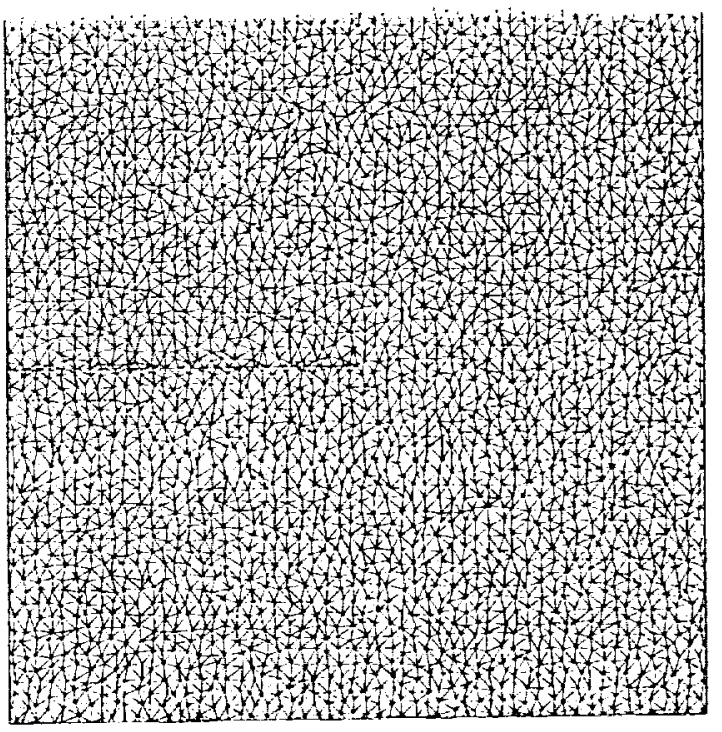

(a)

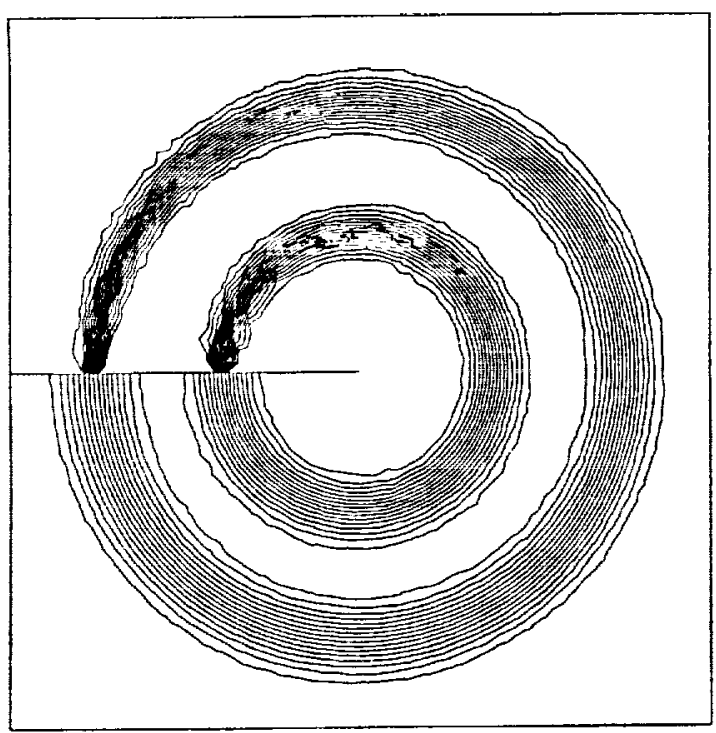

(c)

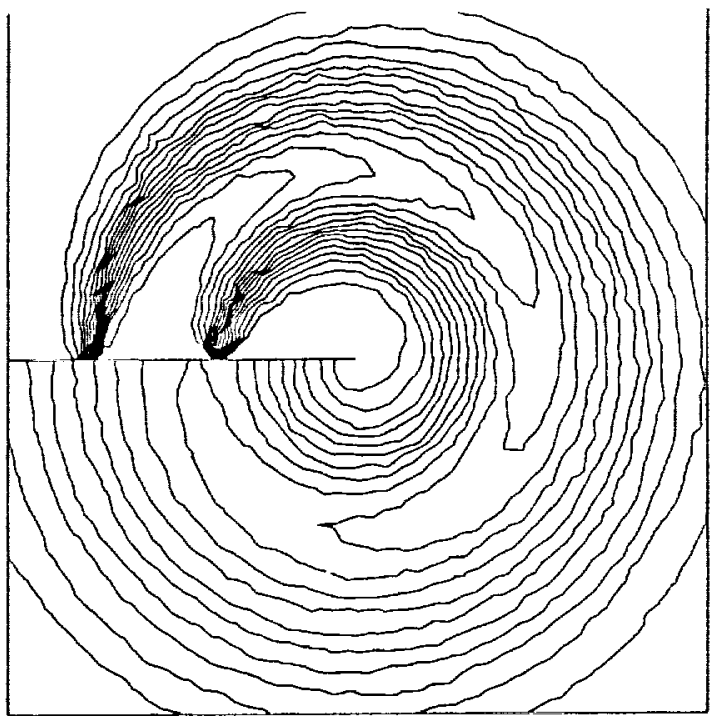

(b)

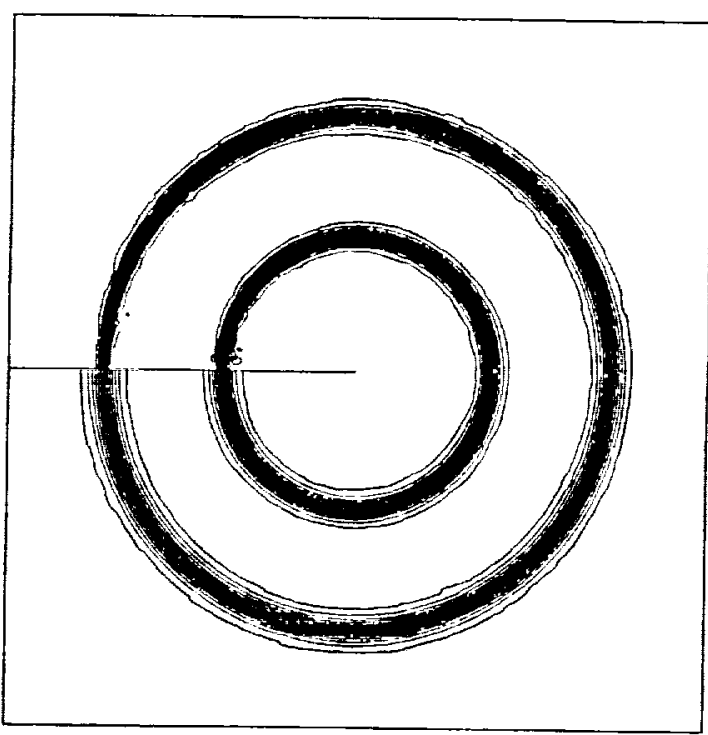

(d)

Figure 8. Comparison of various discretization methods for the circular advection problem [13]. (a) Grid used for the two-dimensional advection equation. (b) Piecewise-constant funite-volume solution. (c) Piecewise-linear finite-volume solution. (d) Piecewise-quadratic finite-rolume solution. 


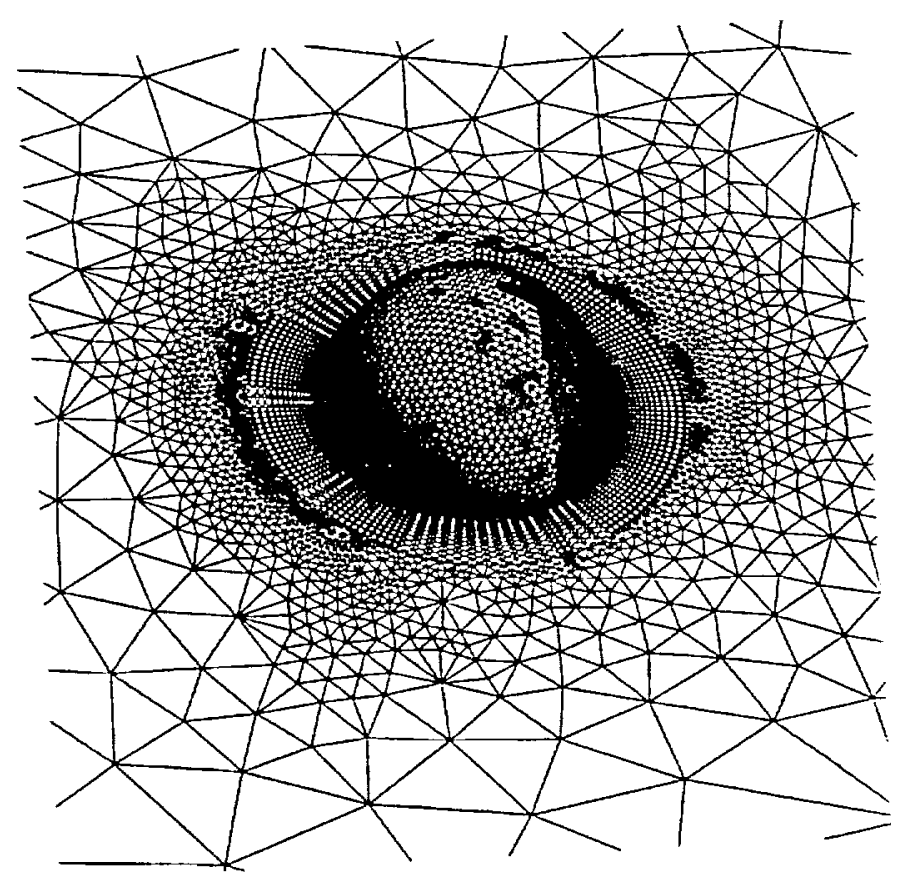

(a)

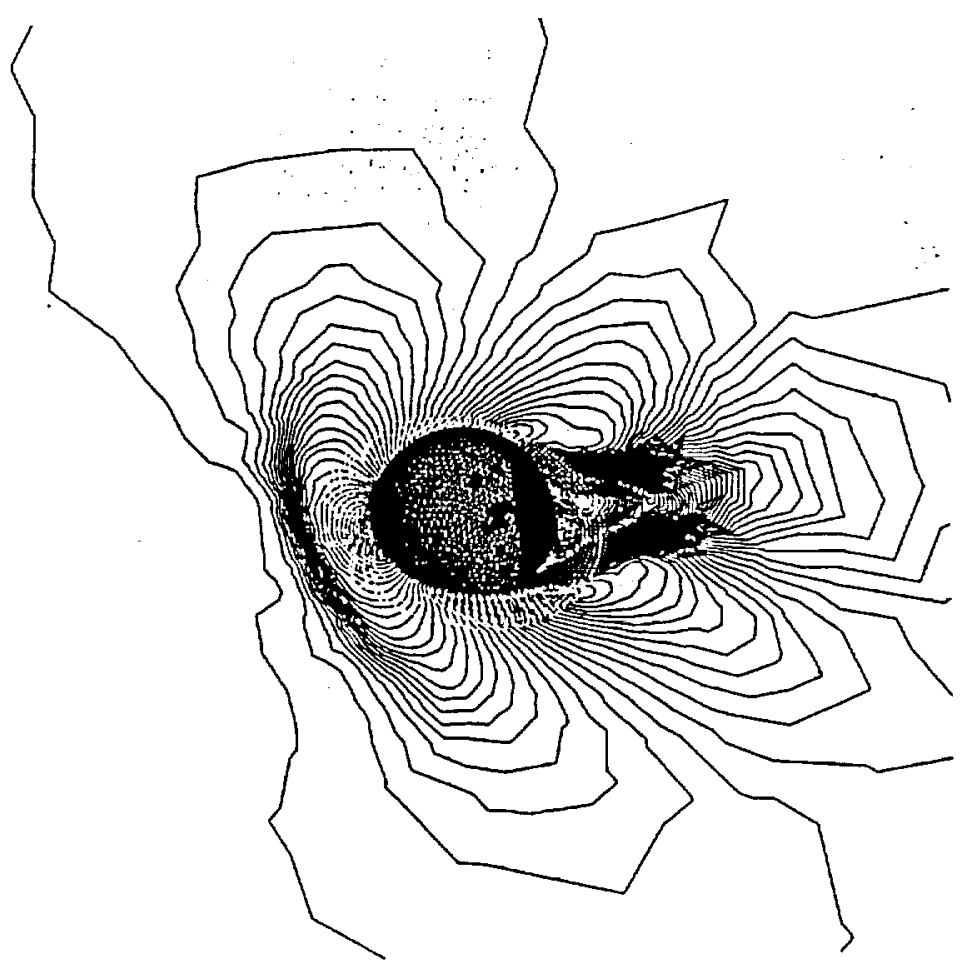

(b)

Figure 9. (a) Adapted hybrid prismatic-tetrahedral grid about a hemisphere. (b) Surface Mach contours on the symmetry plane for supersonic laminar flow $\left(M_{\infty}=1.4, R \epsilon=1000\right)$ [128]. 


\begin{tabular}{|c|c|c|c|}
\hline \multicolumn{3}{|c|}{ REPORT DOCUMENTATION PAGE } & $\begin{array}{l}\text { Form Approved } \\
\text { OMB No 0704-0188 }\end{array}$ \\
\hline \multicolumn{4}{|c|}{ 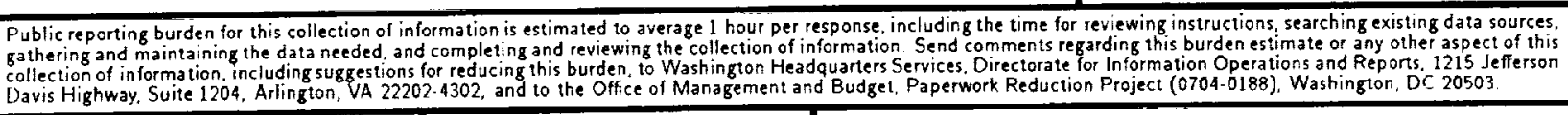 } \\
\hline 1. AGENCY USE ONLY(Leave blank) & \begin{tabular}{|l|l} 
2. REPORT DATE & \\
February 1995 &
\end{tabular} & \multicolumn{2}{|c|}{$\begin{array}{l}\text { 3. REPORT TYPE AND DATES COVERED } \\
\text { Contractor Report }\end{array}$} \\
\hline \multicolumn{3}{|c|}{$\begin{array}{l}\text { 4. TITLE AND SUBTITLE } \\
\text { A PERSPECTIVE ON UNSTRUCTURED GRID FLOW SOLVERS }\end{array}$} & \multirow[t]{2}{*}{$\begin{array}{l}\text { 5. FUNDING NUMBERS } \\
\text { C NAS1-19480 } \\
\text { WU } 505-90-52-01\end{array}$} \\
\hline \multicolumn{3}{|l|}{$\begin{array}{l}\text { 6. AUTHOR(5) } \\
\text { V. Venkatakrishnan }\end{array}$} & \\
\hline \multicolumn{3}{|c|}{$\begin{array}{l}\text { 7. PERFORMING ORGANIZATION NAME(S) AND ADDRESS(ES) } \\
\text { Institute for Computer Applications in Science } \\
\text { and Engineering } \\
\text { Mail Stop 132C, NASA Langley Research Center } \\
\text { Hampton, VA 23681-0001 }\end{array}$} & $\begin{array}{l}\text { 8. PERFORMING ORGANIZATION } \\
\text { REPORT NUMBER } \\
\text { ICASE Report No. } 95-3\end{array}$ \\
\hline \multicolumn{3}{|c|}{$\begin{array}{l}\text { 9. SPONSORING/MONITORING AGENCY NAME(S) AND ADDRESS(ES) } \\
\text { National Aeronautics and Space Administration } \\
\text { Langley Research Center } \\
\text { Hampton, VA } 23681-0001\end{array}$} & $\begin{array}{l}\text { 10. SPONSORING/MONITORING } \\
\text { AGENCY REPORT NUMBER } \\
\text { NASA CR-195025 } \\
\text { ICASE Report No. } 95-3\end{array}$ \\
\hline \multicolumn{4}{|c|}{$\begin{array}{l}\text { 11. SUPPLEMENTARY NOTES } \\
\text { Langley Technical Monitor: Dennis M. Bushnell } \\
\text { Final Report } \\
\text { Submitted to AIAA Journal }\end{array}$} \\
\hline \multicolumn{2}{|c|}{$\begin{array}{l}\text { 12a. DISTRIBUTION/AVAILABILITY STATEMENT } \\
\text { Unclassified-Unlimited } \\
\text { Subject Category } 64\end{array}$} & & 12b. DISTRIBUTION CODE \\
\hline \multicolumn{4}{|c|}{$\begin{array}{l}\text { 13. ABSTRACT (Maximum } 200 \text { words) } \\
\text { This survey paper assesses the status of compressible Euler and Navier-Stokes solvers on unstructured grids. Different } \\
\text { spatial and temporal discretization options for steady and unsteady flows are discussed. The integration of these } \\
\text { componentsinto an overall framework to solve practical problems is addressed. Issues such as grid adaptation, higher } \\
\text { order methods, hybrid discretizations and parallel computing are briefly discussed. Finally, some outstanding issues } \\
\text { and future research directions are presented. }\end{array}$} \\
\hline \multirow{2}{*}{\multicolumn{3}{|c|}{$\begin{array}{l}\text { 14. SUBJECT TERMS } \\
\text { Unstructured; Finite Volume; Finite Element; Adaptive Grids }\end{array}$}} & $\begin{array}{l}\text { 15. NUMBER OF PAGES } \\
39\end{array}$ \\
\hline & & & $\begin{array}{c}\text { 16. PRICE CODE } \\
A 03\end{array}$ \\
\hline $\begin{array}{l}\text { 17. SECURITY CLASSIFICATION } \\
\text { OF REPORT } \\
\text { Unclassified }\end{array}$ & $\begin{array}{l}\text { 18. SECURITY CLASSIFICATION } \\
\text { OF THIS PAGE } \\
\text { Unclassified }\end{array}$ & $\begin{array}{l}\text { 19. SECURITY CLASSIFICATION } \\
\text { OF ABSTRACT }\end{array}$ & $\begin{array}{l}\text { 20. LIMITATION } \\
\text { OF ABSTRACT }\end{array}$ \\
\hline NSN 7540-01-280-5500 & & & $\begin{array}{l}\text { Standard Form 298(Rey. 2-89) } \\
\text { Prescribed by ANSI S1d. Z39-18 } \\
298.102\end{array}$ \\
\hline
\end{tabular}



National Aeronautics and

Space Administration

Langley Research Center

Mail Code 180

Hampton, VA 23681-00001

BULK RATE

POSTAGE \& FEES PAID

NASA

Otficial Business

Penalty for Private Use, $\mathbf{5 3 0 0}$

Permit No. G-27 